\title{
Theory of the Nernst effect near quantum phase transitions in condensed matter and in dyonic black holes
}

\section{Citation}

Hartnoll, Sean A., Pavel K. Kovtun, Markus Müller, and Subir Sachdev. 2007. “Theory of the Nernst Effect near Quantum Phase Transitions in Condensed Matter and in Dyonic Black Holes." Physical Review B 76 (14). https://doi.org/10.1103/physrevb.76.144502.

\section{Permanent link}

http://nrs.harvard.edu/urn-3:HUL.InstRepos:41417250

\section{Terms of Use}

This article was downloaded from Harvard University's DASH repository, and is made available under the terms and conditions applicable to Other Posted Material, as set forth at http:// nrs.harvard.edu/urn-3:HUL.InstRepos:dash.current.terms-of-use\#LAA

\section{Share Your Story}

The Harvard community has made this article openly available. Please share how this access benefits you. Submit a story. 
NSF-KITP-07-144

\title{
Theory of the Nernst effect near quantum phase transitions in condensed matter, and in dyonic black holes
}

\author{
Sean A. Hartnoll, ${ }^{1}$ Pavel K. Kovtun, ${ }^{1}$ Markus Müller, ${ }^{2}$ and Subir Sachdev ${ }^{2}$ \\ ${ }^{1}$ Kavli Institute for Theoretical Physics, \\ University of California, Santa Barbara, CA 93106-4030, USA \\ ${ }^{2}$ Department of Physics, Harvard University, Cambridge MA 02138, USA
}

(Dated: June 2007)

\begin{abstract}
We present a general hydrodynamic theory of transport in the vicinity of superfluid-insulator transitions in two spatial dimensions described by "Lorentz"-invariant quantum critical points. We allow for a weak impurity scattering rate, a magnetic field $B$, and a deviation in the density, $\rho$, from that of the insulator. We show that the frequency-dependent thermal and electric linear response functions, including the Nernst coefficient, are fully determined by a single transport coefficient (a universal electrical conductivity), the impurity scattering rate, and a few thermodynamic state variables. With reasonable estimates for the parameters, our results predict a magnetic field and temperature dependence of the Nernst signal which resembles measurements in the cuprates, including the overall magnitude. Our theory predicts a "hydrodynamic cyclotron mode" which could be observable in ultrapure samples. We also present exact results for the zero frequency transport co-efficients of a supersymmetric conformal field theory (CFT), which is solvable by the AdS/CFT correspondence. This correspondence maps the $\rho$ and $B$ perturbations of the $2+1$ dimensional CFT to electric and magnetic charges of a black hole in the $3+1$ dimensional anti-de Sitter space. These exact results are found to be in full agreement with the general predictions of our hydrodynamic analysis in the appropriate limiting regime. The mapping of the hydrodynamic and AdS/CFT results under particle-vortex duality is also described.
\end{abstract}




\section{INTRODUCTION}

A key indication that the normal state of the cuprate superconductors is aberrant came from the pioneering measurements of the Nernst effect by Ong and collaborators [1-4]. Also of interest here are measurements of the Nernst effect in $\mathrm{Nb}_{0.15} \mathrm{Si}_{0.85}$ films by Behnia and collaborators $[5,6]$. The Nernst co-efficient measures the transverse voltage arising in response to an applied thermal gradient in the presence of a magnetic field. The response of Fermi liquids is associated with a weak particle-hole asymmetry in the spectrum of the fermionic excitations near the Fermi level [7]. The large observed response, and its striking and unexpected dependence on the magnetic field, temperature, and carrier concentration indicated that an explanation starting from a metallic Fermi liquid state could not be tenable. Instead, Ong and collaborators argued that their observations called for a description in terms of a liquid of quantized vortices and anti-vortices in the superconducting order (and its precursors) at low temperatures.

A complete theory of the dynamics of the vortex liquid state is so far lacking. Ussishkin et al. [8] used a classical Gaussian theory of superconducting fluctuations, and Mukerjee and Huse [9] extended this to a time-dependent Ginzburg-Landau model. Podolsky et al. [10] applied classical Langevin equations to a model of phase variables residing on the sites of a hypothetical lattice. Anderson [11] has taken a speculative view of the vortex liquid, arguing against the conventional Debye-screening of vortex interactions. While some experimental trends are successfully described by Refs. 8-10, it would be useful to have a kinematic approach which is also able to include quantum effects, and extends across the superfluidinsulator transition. Quantum effects which surely play an more important role at lower temperatures, especially in the underdoped region. Indeed, it is the equal importance of thermal and quantum fluctuations which underlies the difficulty in describing this vortex liquid.

As in the recent work by Bhaseen, Green, and Sondhi [12], this paper will advocate an approach departing from the quantum critical region of a zero temperature $(T)$ quantum phase transition between a superconductor and an insulator. This is the region where the primary perturbation from the physics of the $T=0$ quantum critical point is the

temperature. The single energy scale, $k_{B} T$, then determines observable properties, including the values of diffusion constants and transport co-efficients, in a manner that has been discussed at length elsewhere $[13,14]$. The electrical conductivity of this quantum critical system, which we denote $\sigma_{Q}$, will play a prominent role in our results. In $2+1$ dimensions, near quantum critical points which obey hyperscaling properties, this conductivity is given by $[15-17]$

$$
\sigma_{Q}=\frac{4 e^{2}}{h} \Phi_{\sigma}
$$

where in the quantum critical region $\Phi_{\sigma}$ is a universal dimensionless number dependent only upon the universality class of the critical point. 


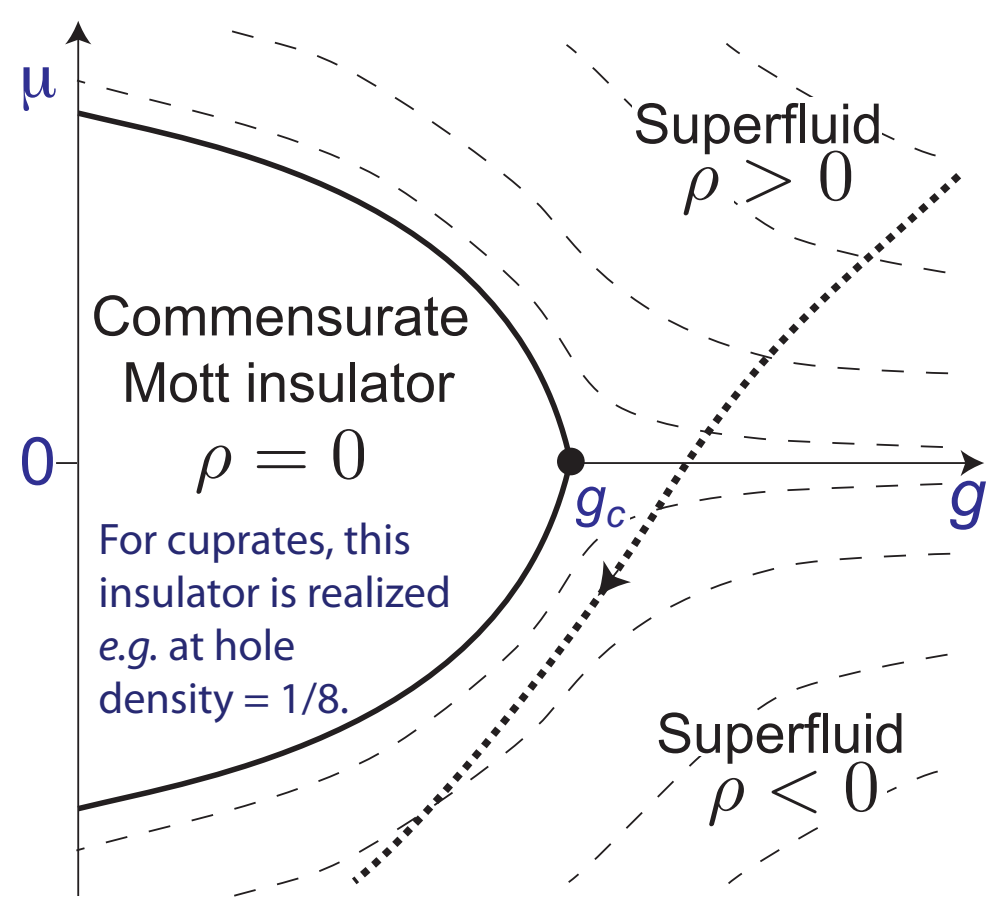

FIG. 1: Zero temperature $(T=0)$, zero field $(B=0)$ phase diagram in the vicinity of the quantum critical point described by the CFT, represented by the filled circle. The coupling $g$ represents a parameter which tunes between a superfluid and a Mott insulator which is at a density commensurate with the underlying lattice. The chemical potential $\mu$ introduces variations in the density and $\rho$ is difference in the density of pairs of holes in the superfluid from that in the Mott insulator. The thin dashed lines are contours of constant $\rho$. In the application to the cuprate superconductors, the Mott insulator with $\rho=0$ could be, e.g., an insulating state at hole density $\delta_{I}=1 / 8$ in a generalized phase diagram; then $\rho=\left(\delta-\delta_{I}\right) /\left(2 a^{2}\right)$, where $a$ is the lattice spacing. The thick dotted line represents a possible trajectory of a particular compound as its hole density is decreased; note that the ground state is always a superconductor along this trajectory, even at $\delta=1 / 8$ (although there will be a dip in $T_{c}$ near $\delta=1 / 8$ as is also clear from Fig. 2). Note that the parent Mott insulator with zero hole density is not shown above. This paper will describe electrical and thermal transport in the above phase diagram perturbed by an applied magnetic field $B$ and a small density of impurities.

The discussion so far applies, strictly speaking, only to systems which are exactly at the commensurate density for which a gapped Mott insulator can form. The cuprates, and other experimental systems, are not generically at these special densities, and so it is crucial to develop a theory that is applicable at generic densities. Such a theory will emerge as a special case of our more general results below. We allow the density to take values $\rho$ by applying a chemical potential $\mu$, as shown in Figs. 1 and 2 .

We emphasize that $\rho$ measures the deviation in particle number density from the density of the commensurate insulator $[19,20]$; so $\rho$ can be positive or negative, and we will see 


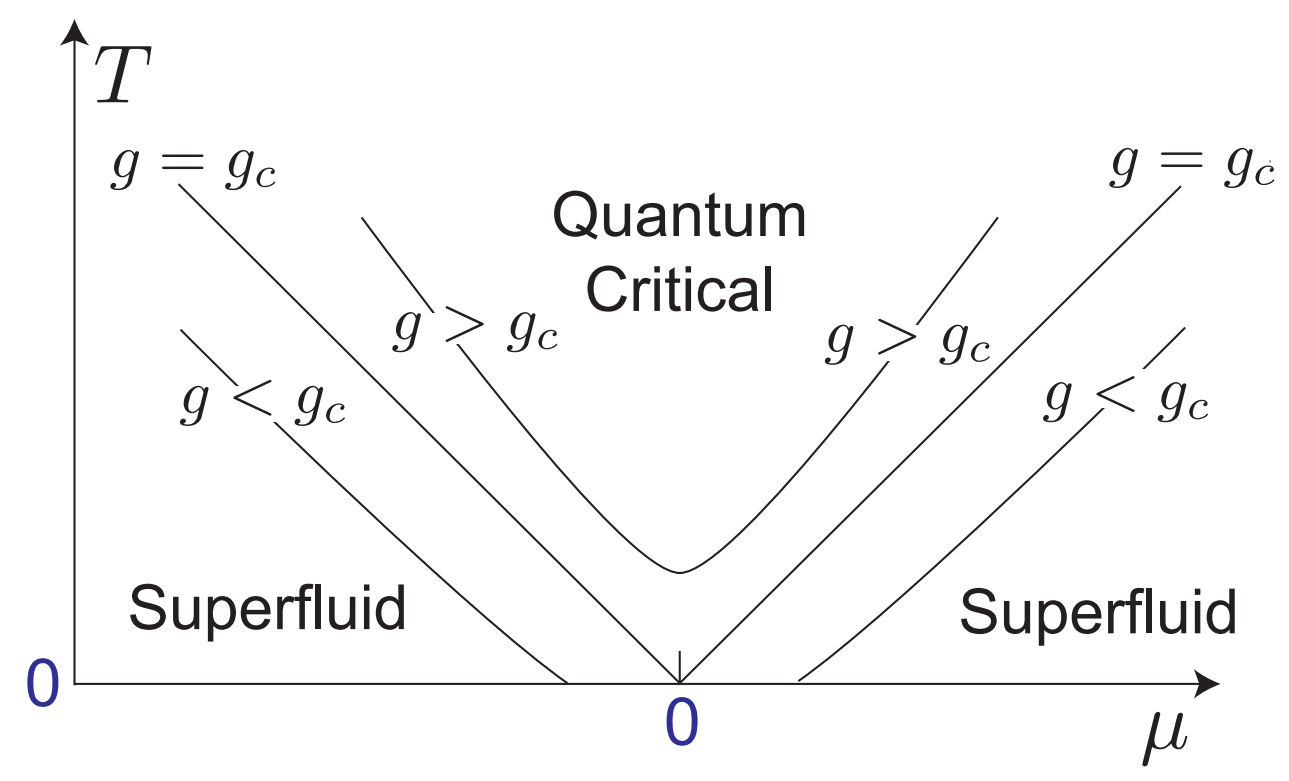

FIG. 2: Nonzero temperature $(T)$ phase diagram at $B=0$ along three vertical cuts (i.e. fixed $g$ ) in Fig. 1. The lines indicate Kosterlitz-Thouless phase transitions at $T=T_{K T}$ associated with the loss of superfluid order as a function of $\mu$ for different values of $g$. At $g=g_{c}, T_{K T} /|\mu|$ is a universal number determined by the CFT at $g=g_{c}, \mu=0$ [18]. This paper describes transport properties in the non-superfluid region above $T_{K T}$, in the presence of an applied magnetic field $B$ and a small impurity scattering $\tau_{\text {imp }}$. The results of the supersymmetric CFT solvable by AdS/CFT in Section $\mathrm{V}$ are limited to $g=g_{c}$, but allow arbitrary variations in $\mu$ and $B$ away from quantum criticality as long there is no phase transition into a superfluid (or other) state.

that the sign of $\rho$ determines the sign of the Hall resistivity and other transport coefficients. Also, purely as a choice of convention, we will measure $\rho$ in terms of density of pairs of holes; this choice does not imply the degrees of freedom of the underlying theory are Cooper pairs, although this is the case in the simplest model. We will use general hydrodynamic arguments (specialized to "relativistic" quantum critical points) to show that the frequency $(\omega)$ dependent conductivity, $\sigma_{x x}$ at a generic density $\rho$ is given by

$$
\sigma_{x x}=\sigma_{Q}+\frac{4 e^{2} \rho^{2} v^{2}}{(\varepsilon+P)} \frac{1}{\left(-i \omega+1 / \tau_{\mathrm{imp}}\right)}
$$

where the system is characterized by the thermodynamic state variables $\varepsilon$, the energy density, and $P$, the pressure-we will specify their temperature dependencies shortly in Eq. (1.8). The factor of $(2 e)^{2}$ is a consequence of our choice for the normalization of $\rho$; note that product $2 e \rho$ measures the net charge density, and so is independent of this convention. We assume there is a dilute concentration of impurities which relax the conserved momentum ${ }^{1}$, and lead to the scattering rate $1 / \tau_{\text {imp }}$ - the temperature dependence of $\tau_{\text {imp }}$ is specified in Eq. (1.11).

\footnotetext{
${ }^{1}$ Umklapp scattering can also lead to momentum relaxation, but this is exponentially small at low $T$.
} 
The parameter $v$ is a velocity characteristic of the quantum critical point which is assumed to have dynamic critical exponent $z=1$. Finally, the crucial parameter $\sigma_{Q}$, is the same quantum conductance which appeared in Eq. (1.1). However, as one moves away from the critical coupling $g=g_{c}$ and adds a non-zero $\mu$ in Fig. 2, $\Phi_{\sigma}$ will acquire a dependence upon the ratios $\left(g-g_{c}\right) / T^{1 / \nu}$ and $\mu / T$ which can be included unchanged in our results below (here $\nu$ is the usual correlation length exponent).

It is perhaps helpful to note here the "non-relativistic" limit of Eq. (1.2), which does not

constitute the regime of primary interest of this paper. In this limit $\varepsilon+P \approx|\rho| m v^{2}$ (where $m$ is the mass of the particles), and then the second term takes the form of the conventional Drude result.

To develop a theory for the Nernst effect, we need to apply a magnetic field $B$ to the system described so far. A central result of this paper is that for not too strong $B$ fields, the Nernst response, and a set of related thermoelectric transport co-efficients, are completely determined by the thermodynamic variables and impurity scattering rate appearing in Eq. (1.2) and the single universal transport co-efficient $\sigma_{Q}$. In particular, no additional transport co-efficients are needed. Thus there are a large number of Wiedemann-Franz-like relations which relate all the thermoelectric response functions to the regular part of the electrical conductivity in zero field, $\sigma_{Q}$. We will also determine the frequency dependence of these transport co-efficients; explicit expressions are given below.

In their work, Bhaseen et al. [12] only considered a non-zero $B$, with $\rho=1 / \tau_{\text {imp }}=$ 0 . Their primary new result concerned the longitudinal thermal conductivity, $\kappa_{x x}$ at zero frequency. Our result for $\kappa_{x x}(\omega=0)$ is consistent with theirs, and further, we show further that it is related to $\sigma_{Q}$ by a Wiedemann-Franz like identity (Eq. (1.28) below). However, remarkably, unlike the conventional identity which specifies the ratio of $\kappa_{x x}$ to the electrical conductivity, our identity specifies the ratio of $\kappa_{x x}$ to the electrical resistivity. This suggests a physical picture of transport currents carried by vortices rather than particles, at least when the perturbation associated with $B$ is larger than that associated with $\rho$.

\section{A. Characterization of systems under consideration}

Let us now specify the class of theories to which our results apply. Current theories of the superfluid-insulator transition in non-random systems in $2+1$ dimensions are described by quantum field theories which are Lorentz invariant, and are therefore conformal field theories (CFTs). Consequently, we will mainly restrict our attention here to $T>0$ "quantum critical" phases of CFTs, and the general structure of their response to a non-zero $\rho$ (which is not restricted to be small) and small $B$ and $1 / \tau_{\text {imp }}$. We expect that many of our results, and especially the magnetohydrodynamic analysis in Section III, can be generalized to a wider class of systems, but we will not discuss such a generalization here.

Specific examples of CFTs to which our results apply are: 
(i) The superfluid-insulator transition of the boson Hubbard model on a two-dimensional lattice with a density of an integer number of bosons per unit cell. The bosons carry charge $\pm 2 e$ because they are caricatures of Cooper pairs. The critical point is described [21] by the Wilson-Fisher fixed point of the $|\psi|^{4}$ field theory of a complex scalar $\psi$ (representing the boson annihilation operator), see Eq. (1.3) below. This field theory also has a dual representation [22, 23] in terms of a vortex field $\varphi$ coupled to an emergent $\mathrm{U}(1)$ gauge field. Our results apply equally to both representations, and the observable properties do not depend, naturally, on whether the particle or vortex representation is used to describe the CFT.

(ii) The superfluid-insulator transition of the boson Hubbard model on a two-dimensional lattice with a mean density of a rational number, $p / q$ (with $p, q$, co-prime integers), of bosons per unit cell. A 'deconfined' critical point [24] is then possible [19, 25] between the superfluid and an insulator with valence-bond-solid order and is described by the theory of $q$ flavors of vortex fields $\varphi_{\ell}$ coupled to an emergent U(1) gauge field. This field theory can also be 'undualized' to a "quiver gauge theory" of fractionalized bosons with charge $\pm 2 e / q$ [19].

(iii) Electronic models with a $d$-wave superconducting ground state can also undergo deconfined phase transitions to insulating states with valence-bond-solid order [26, 27]. The CFTs of these transitions have Dirac fermion degrees of freedom, representing the gapless, Bogoliubov quasiparticle excitations of the $d$-wave superconductor, in addition to the multiple vortex and gauge fields found above in (ii).

(iv) Yang-Mills gauge theories with a $\mathrm{SU}(N)$ gauge group and $\mathcal{N}=8$ supersymmetry. These are attracted in the infrared to a superconformal field theory (SCFT) which is solvable in the large $N$ limit via the AdS/CFT mapping. This solution has been used in previous work $[14,28]$ to obtain the collisionless-to-hydrodynamic crossover in the transport of a conserved $\mathrm{SO}(8) \mathrm{R}$-charge, as well as an exact value for $\Phi_{\sigma}$. Here we will examine, as in other recent work [29], the deformation of the SCFT by a non-zero $B$ and $\rho$. The $B$ field and density $\rho$ are both associated with a $\mathrm{U}(1)$ subgroup of the $\mathrm{SO}(8) \mathrm{R}$ charge. After the AdS/CFT mapping, $B$ and $\rho$ correspond to the magnetic and the electric charge of a black hole in AdS space. We will present exact results for the conserved current correlators of the dyonic black hole in Section V, which allows us to obtain corresponding exact results for the Nernst and related thermoelectric responses of the SCFT. In the appropriate hydrodynamic limit, these results are found to be in full agreement with the more general magnetohydrodynamic analysis in Section III. Additional comparisons between the hydrodynamic and AdS/CFT results appear in a separate paper [30].

It is worth reiterating that not all of the above CFTs are purely bosonic, and the examples in ( $i i i)$ and $(i v)$ contain fermionic degrees of freedom. Furthermore, in cases (ii) and (iii), the bosonic degrees of freedom of the CFT are not Cooper pairs, but fractions of a Cooper pair with charges determined by the density of the Mott insulator. 


\section{B. A simple model}

Before presenting our main results, it is useful to establish notation by explicitly writing down the simplest of the CFTs listed above. This is the $|\psi|^{4}$ field theory for bosons with charge $\pm 2 e$ and action

$$
\mathcal{S}=\int d^{2} r d \tau\left[\left|\left(\partial_{\tau}-i \frac{2 e}{\hbar} A_{\tau}\right) \psi\right|^{2}+v^{2}\left|\left(\vec{\nabla}-i \frac{2 e}{\hbar c} \vec{A}\right) \psi\right|^{2}-g|\psi|^{2}+\frac{u}{2}|\psi|^{4}\right]
$$

where $\vec{r}=(x, y)$ is a 2-dimensional spatial co-ordinate, $\tau$ is imaginary time, $g$ is the coupling which tunes the system from the superfluid to the insulator (see Figs. 1,2), and the quartic coupling $u$ is attracted to the Wilson-Fisher fixed point value in the infrared limit associated with the CFT. The electromagnetic gauge potential $A_{\mu}$ is non-fluctuating (and is not to be confused with the emergent $\mathrm{U}(1)$ gauge field of the vortex CFTs noted above). Its timecomponent takes an imaginary value (in imaginary time) which determines the chemical potential

$$
i 2 e A_{\tau}=\mu
$$

while the spatial components take $\tau$-independent values so that

$$
\vec{\nabla} \times \vec{A}=B
$$

with a spacetime-independent magnetic field $B$. The density, $\rho$, is defined, as usual, by the derivative of the partition function with respect to the chemical potential

$$
\rho=\frac{k_{B} T}{\hbar \mathcal{V}}\left\langle\frac{\partial \mathcal{S}}{\partial \mu}\right\rangle
$$

where $\mathcal{V}$ is the volume of the system. We reiterate that $\rho$ measures the difference in the density from that of the commensurate, $T=0$, insulating state, and not the total density. Also, $\rho$ is a charge density in the sense that it measures the number density of particles minus the number density of anti-particles.

Another parameter above which will be important for experimental comparisons is the velocity $v$. Note that it plays the role of the velocity of "light" in the "relativistic" CFT. It is determined here by the parameters of the underlying boson Hubbard (or other microscopic) model whose superfluid-insulator transition is described by the above CFT. It is important to distinguish $v$ from the velocity $c$, which is the actual velocity of light. Here $c$ merely plays the role of a coupling constant which relates the value of $B$ to physical CGS units, and is not a velocity associated with the dynamics of the physical model under consideration. Because $v \ll c$, we can neglect the actual relativistic corrections associated with the physical quantum fluctuations of the photon field $A_{\mu}$.

With the definition of $v$ at hand, we can now begin comparing the various energy scales which characterize the system. The largest energy scales which characterize the deviation 
from the $T=0$ quantum critical point are $k_{B} T$, an energy scale $m_{0} \sim\left|g-g_{c}\right|^{\nu}$ associated with the deviation from critical coupling, and the chemical potential $\mu$. We will generally assume that $k_{B} T$ is the largest of these scales; our results allow $m_{0}$ and $\mu$ to be of order $k_{B} T$, but not too much larger. For the solvable SCFT theories considered in Section V, the energy scales associated with $\rho$ and $B$ will not be restricted to small values. However, for the more general analysis in the remainder of the paper which applies also to non-supersymmetric CFTs, we will assume that the perturbation due to $B$ is small, and in particular,

$$
\hbar v \sqrt{2 e B /(\hbar c)} \ll k_{B} T .
$$

Some thermodynamic state variables will also appear in our transport result. Their temperature dependencies obey scaling forms similar to those computed earlier for the present theory at $\rho=0$ in Ref. 31, and for $\rho \neq 0$ in Ref. 32. In particular, we will need results for the energy density, $\varepsilon$, and the pressure, $P$ which obey

$$
\begin{aligned}
\varepsilon & =k_{B} T\left(\frac{k_{B} T}{\hbar v}\right)^{2} \Phi_{\varepsilon}, \\
P & =k_{B} T\left(\frac{k_{B} T}{\hbar v}\right)^{2} \Phi_{P}
\end{aligned}
$$

where, as in Eq. (1.1), $\Phi_{\varepsilon, P}$ are dimensionless universal numbers which depend on the ratios $\left(g-g_{c}\right) /\left(k_{B} T\right)^{1 / \nu}$ and $\mu /\left(k_{B} T\right)[31,32]$. The dependence on $B$ is not difficult to account for, but will be subdominant, and non-singular, under the condition in Eq. (1.7).

The final parameter to introduce in our theory of the Nernst effect and other thermoelectric response functions is the momentum relaxation rate $1 / \tau_{\text {imp }}$. The theory defined so far conserves total momentum, and thus, such relaxation requires an additional perturbation. We assume that the relaxation is caused by a weak random impurity potential $V(r)$ which couples to the most relevant perturbation allowed by symmetry at the CFT fixed point. For the present theory, this is the operator $|\psi|^{2}$, and therefore the impurity action is

$$
\mathcal{S}_{\text {imp }}=\int d \tau \int d^{2} r V(r)|\psi(r, \tau)|^{2}
$$

We will take a "quenched" average over the ensemble of impurity potentials which obey

$$
\overline{V(r)}=0 ; \overline{V(r) V\left(r^{\prime}\right)}=V_{\mathrm{imp}}^{2} \delta^{2}\left(r-r^{\prime}\right),
$$

and work to order $V_{\mathrm{imp}}^{2}$. Note that total energy and charge are conserved in the presence of $V(r)$, and momentum is the only conserved quantity which will relax. We estimate the momentum relaxation rate in Section IV and find

$$
\frac{1}{\tau_{\mathrm{imp}}} \sim V_{\mathrm{imp}}^{2} T^{3-2 / \nu} .
$$

The condition for this scattering to be small is

$$
\hbar / \tau_{\text {imp }} \ll k_{B} T \text {. }
$$


The present model has $\nu \approx 2 / 3$, and so $1 / \tau_{\text {imp }}$ depends upon temperature only very weakly. Indeed, all the CFTs noted earlier are expected to have a similar value for $\nu$. It is therefore a reasonable first approximation to treat $1 / \tau_{\mathrm{imp}}$ as a temperature-independent constant. We will also ignore the dependence of $1 / \tau_{\text {imp }}$ on $B$ and $\rho$, under the condition in Eq. (1.7).

\section{Summary of results for the thermo-electric response}

We finally turn to a statement of our main results for the transport co-efficients. We are interested in the response of the electrical current $\vec{J}$ and the heat current $\vec{Q}$ to an applied electric field $\vec{E}$ and a temperature gradient $\vec{\nabla} T$. The precise definitions of $\vec{J}$ and $\vec{Q}$ appear in the contexts of the models studied in the body of the paper. The electric field can be applied by allowing for a weak spatial dependence in the chemical potential $\mu$ (which is then, formally, the electrochemical potential) with $2 e \vec{E}=-\vec{\nabla} \mu$, while the temperature gradient describes a similar weak spatial dependence in $T$. The transport co-efficients are defined by the relation

$$
\left(\begin{array}{c}
\vec{J} \\
\vec{Q}
\end{array}\right)=\left(\begin{array}{cc}
\hat{\sigma} & \hat{\alpha} \\
T \hat{\alpha} & \hat{\kappa}
\end{array}\right)\left(\begin{array}{c}
\vec{E} \\
-\vec{\nabla} T
\end{array}\right),
$$

where $\hat{\sigma}, \hat{\alpha}$ and $\hat{\kappa}$ are $2 \times 2$ matrices acting on the spatial indices $x, y$. Rotational invariance in the plane imposes the form

$$
\hat{\sigma}=\sigma_{x x} \hat{1}+\sigma_{x y} \hat{\epsilon},
$$

where $\hat{1}$ is the identity, and $\hat{\epsilon}$ is the antisymmetric tensor $\hat{\epsilon}_{x y}=-\hat{\epsilon}_{y x}=1 . \quad \sigma_{x x}$ and $\sigma_{x y}$ describe the longitudinal and Hall conductivity, respectively. An analogous form holds for the thermoelectric conductivity $\hat{\alpha}$ (which determines the Peltier, Seebeck, and Nernst effects), as well as for the matrix $\hat{\bar{\kappa}}$ which governs thermal transport in the absence of electric fields. The latter applies to samples connected to conducting leads, allowing for a stationary current flow. In contrast, the thermal conductivity, $\hat{\kappa}$, is defined as the heat current response to $-\vec{\nabla} T$ in the absence of an electric current (electrically isolated boundaries). It is given by

$$
\hat{\kappa}=\hat{\kappa}-T \hat{\alpha} \hat{\sigma}^{-1} \hat{\alpha} .
$$

Finally, the Nernst response is defined as the electric field induced by a thermal gradient in the absence of an electric current, and is given in linear response by the relation $\vec{E}=-\hat{\vartheta} \vec{\nabla} T$, with

$$
\hat{\vartheta}=-\hat{\sigma}^{-1} \hat{\alpha} .
$$

The Nernst signal is the transverse response, $e_{N} \equiv \vartheta_{y x}$. The Nernst co-efficient is usually defined as $\nu=e_{N} / B$, which tends to become field independent at small $B$. The Nernst signal is expected to be positive if it is due to driven vortices, while it is generally negative if it arises from quasiparticle excitations [33]. 
We now present our main results for the transport co-efficients. For the computations using AdS/CFT applied to the SYM theory in Section V, results can be obtained for general external frequency, $\omega$. However, our more general hydrodynamic results apply only for $\hbar \omega \ll k_{B} T$, and this condition is assumed in the remainder of this section. We begin by presenting our complete result for the frequency dependence of the longitudinal electrical conductivity (whose $B \rightarrow 0$ limit was already quoted in Eq. (1.2)):

$$
\sigma_{x x}=\sigma_{Q}\left[\frac{\left(\omega+i / \tau_{\mathrm{imp}}\right)\left(\omega+i \gamma+i \omega_{c}^{2} / \gamma+i / \tau_{\mathrm{imp}}\right)}{\left(\omega+i \gamma+i / \tau_{\mathrm{imp}}\right)^{2}-\omega_{c}^{2}}\right] .
$$

The overall scale is set by the quantum conductance $\sigma_{Q}$ introduced in Eq. (1.2), and the remainder depends upon two important frequency scales which will appear throughout our analysis. These frequencies are

$$
\omega_{c} \equiv \frac{2 e B \rho v^{2}}{c(\varepsilon+P)}
$$

and

$$
\gamma \equiv \frac{\sigma_{Q} B^{2} v^{2}}{c^{2}(\varepsilon+P)}
$$

We identify the first frequency, $\omega_{c}$, as a cyclotron frequency. This seems a natural interpretation in view of the damped resonance present in the denominator of Eq. (1.17). Note that in the non- relativistic limit where $\varepsilon+P \approx|\rho| m v^{2}, \omega_{c}$ reduces to the familiar result $\omega_{c}=2 e B /(m c)$. For relativistic particles the cyclotron frequency decreases with the energy $E$ as $\omega_{c}(E)=2 e B c / E$. In the present context where $v$ plays the role of the velocity of light, this is modified to $\omega_{c}(E)=2 e B / c \cdot v^{2} / E$. The hydrodynamic expression (1.18) can be regarded as a thermal average over cyclotron frequencies $\omega_{c}(E \sim T)$, while the proportionality to the charge density, $\omega_{c} \sim \rho$, reflects the fact that particles and antiparticles circle in opposite senses.

We can consider the cyclotron mode as arising either from the motion of particles and anti-particles, or from the motion of vortices and anti-vortices. In the latter interpretation, the roles of $B$ and $\rho$ are interchanged, whilst the expression for $\omega_{c}$ remains invariant. We will have more to say about this 'dual' interpretation in the body of the paper, and further results appear in a separate paper [30].

The second frequency, $\gamma$, is the damping frequency of the cyclotron mode of particles and anti-particles. Note that this damping is present even in the absence of external impurities, and is a consequence of collisions between particles and anti-particles which are executing cyclotron orbits in opposite directions. This should be contrasted from the behavior of a Galilean-invariant system (i.e., a system with no anti-particle excitations) for which Kohn's theorem [34] guarantees an infinitely sharp cyclotron mode. The sharpness of the cyclotron resonance is determined by the ratio

$$
\frac{\gamma}{\omega_{c}}=\Phi_{\sigma} \frac{B}{\phi_{0} \rho}
$$


which up to the factor $\Phi_{\sigma}$ equals the number of flux quanta,

$$
\phi_{0}=\frac{h c}{2 e}
$$

applied per charge $2 e$.

We will see later that a different frequency plays the role of the damping of the cyclotron mode when it is interpreted as due to the motion of vortices and anti-vortices. In that case the damping frequency is

$$
\gamma_{v}=\frac{\omega_{c}^{2}}{\gamma}=\frac{4 e^{2} \rho^{2} v^{2}}{\sigma_{Q}(\varepsilon+P)} .
$$

There is an obvious 'dual' structure apparent upon comparing Eq. (1.19) and (1.22), which we will discuss in more detail. Note that the cyclotron resonance will be visible only in ultrapure samples where $1 / \tau_{\text {imp }} \ll \omega_{c}$. In this case, the cyclotron resonance is sharp in the thermoelectric response associated with particle transport if $\gamma / \omega_{c}=B /\left(\phi_{0} \rho\right) \ll 1$, while the same condition implies a washed out resonance in the dual response functions associated with vortices. In the opposite regime, $\gamma / \omega_{c} \gg 1$, the vortex response, and in particular the Nernst effect, should exhibit a sharp cyclotron resonance in ultrapure samples.

Another notable feature of Eq. (1.17) is the singular nature of the limit associated with the small perturbations of the quantum critical region of Fig. 2. In particular, note that for the d.c. conductivity at $\omega=0$, the value of $\sigma_{x x}$ depends upon the order of limits of $\rho \rightarrow 0$, $B \rightarrow 0$, and $1 / \tau_{\text {imp }} \rightarrow 0$. This singular limit reflects the fact that the low frequency transport studied previously at $\rho=B=0$ in Refs. 14, 17 has ballistic energy propagation and an infinite thermal conductivity. For non-zero $\rho$ or $B$, the energy and number currents can mix with each other, leading to a finite thermal conductivity and an order unity correction to $\sigma_{x x}$, as anticipated for the case $\rho=0$ in Ref. 12 .

A useful property of Eq. (1.17) is that it depends only upon the combination $\omega+i / \tau_{\text {imp }}$. This is actually a property of the long distance limit of the hydrodynamic equations presented in Section III, and is obeyed by all the transport co-efficients. The remainder of this section will therefore present results only in the d.c. limit, while the $\omega$ dependence can be easily reconstructed by replacing $1 / \tau_{\text {imp }} \rightarrow 1 / \tau_{\text {imp }}-i \omega$ (as long as $\hbar \omega \ll k_{B} T$ ).

\section{Nernst effect}

Our central result for the Nernst signal is

$$
\begin{aligned}
e_{N}=\vartheta_{y x} & =\left(\frac{k_{B}}{2 e}\right)\left(\frac{\varepsilon+P}{k_{B} T \rho}\right)\left[\frac{\omega_{c} / \tau_{\mathrm{imp}}}{\left(\omega_{c}^{2} / \gamma+1 / \tau_{\mathrm{imp}}\right)^{2}+\omega_{c}^{2}}\right] \\
& =\frac{1}{\Phi_{\sigma}}\left(\frac{k_{B}}{2 e}\right)\left(\frac{\varepsilon+P}{k_{B} T B / \phi_{0}}\right)\left[\frac{\gamma / \tau_{\mathrm{imp}}}{\left(\omega_{c}^{2} / \gamma+1 / \tau_{\mathrm{imp}}\right)^{2}+\omega_{c}^{2}}\right],
\end{aligned}
$$

where $\Phi_{\sigma}$ is the dimensionless universal number appearing in the expression for the conductivity $\sigma_{Q}$ in Eq. (1.1). We have expressed the Nernst signal in terms of its quantized 
unit,

$$
\frac{k_{B}}{2 e}=43.086 \mu \mathrm{V} / \mathrm{K}
$$

times dimensionless ratios in the various brackets. We can use the relation $\varepsilon+P \approx T s$, where $s$ is the entropy density (see Eq. (3.15)), valid for small $\mu, \rho$, to identify the factor in the second brackets as approximately the entropy per particle in Eq. (1.23), and as the entropy per vortex in Eq. (1.24). The combination of Eqs. (1.1), (1.8), (1.11) and (1.23,1.24) now implies an interesting and non-trivial dependence of the Nernst signal on $B$ and $T$. Those should be observable in experimental regimes where the entire thermoelectric response is dominated by critical superconducting fluctuations, as will be discussed further in Section II.

\section{E. Other thermo-electric transport coefficients}

We conclude this introductory section by mentioning two other results for transport co-efficients whose limiting forms can be compared with earlier computations. For the transverse thermo-electric conductivity we obtain

$$
\alpha_{x y}=\left(\frac{2 e k_{B}}{h}\right)\left(\frac{s / k_{B}}{B / \phi_{0}}\right)\left[\frac{\gamma^{2}+\omega_{c}^{2}+\gamma / \tau_{\mathrm{imp}}(1-\mu \rho /(T s))}{\left(\gamma+1 / \tau_{\mathrm{imp}}\right)^{2}+\omega_{c}^{2}}\right]
$$

While in most recent experiments, the electric conductivity $\hat{\sigma}$ receives the largest contribution from non-critical carriers, the thermoelectric conductivity is dominated by superconducting fluctuations, even far above $T_{c}$. It is thus the main quantity to be compared with recent experimental observations in Section II. As with earlier results, $\alpha_{x y}$ has been written in terms of the quantum unit of the thermoelectric co-efficient $[5,10], 2 e k_{B} / h=6.7 \mathrm{nA} / \mathrm{K}$, times dimensionless ratios. In the absence of impurity scattering, $1 / \tau_{\text {imp }} \rightarrow 0$, the factor in the square brackets is unity, and we have $\alpha_{x y}=s c / B$, a result obtained long ago for noninteracting fermions [40-42] and later derived by Cooper et al. [43] for interacting fermions, and by Bhaseen et al. [12] for the superfluid-insulator transition.

For the longitudinal thermal conductivity we obtain

$$
\begin{aligned}
\kappa_{x x} & =\Phi_{\sigma}\left(\frac{k_{B}^{2} T}{h}\right)\left(\frac{\varepsilon+P}{k_{B} T \rho}\right)^{2}\left[\frac{\left(\omega_{c}^{2} / \gamma\right)\left(\omega_{c}^{2} / \gamma+1 / \tau_{\text {imp }}\right)}{\left(\omega_{c}^{2} / \gamma+1 / \tau_{\text {imp }}\right)^{2}+\omega_{c}^{2}}\right] \\
& =\frac{1}{\Phi_{\sigma}}\left(\frac{k_{B}^{2} T}{h}\right)\left(\frac{\varepsilon+P}{k_{B} T B / \phi_{0}}\right)^{2}\left[\frac{\gamma\left(\omega_{c}^{2} / \gamma+1 / \tau_{\text {imp }}\right)}{\left(\omega_{c}^{2} / \gamma+1 / \tau_{\text {imp }}\right)^{2}+\omega_{c}^{2}}\right],
\end{aligned}
$$

where now $k_{B}^{2} T / h$ is the quantum unit of thermal conductance. In the limit $1 / \tau_{\text {imp }} \rightarrow 0$ and $B \rightarrow 0$, the factor within the square brackets in Eq. (1.27) reduces to unity. The resulting expression for $\kappa_{x x}$ relates it to $\sigma_{Q}$ in a Wiedemann-Franz-like relation, as has been noted by Landau and Lifshitz [46] (and elaborated on recently in Ref. 47). This relation suggests a physical picture of transport due to particles/anti-particles carrying charges $\pm 2 e$ and entropy per particle $s / \rho$. 
In the complementary limit of $1 / \tau_{\text {imp }} \rightarrow 0$ and $\rho \rightarrow 0$ the factor within the square brackets in Eq. (1.28) reduces to unity. Now $\kappa_{x x}$ is proportional to the resistivity $1 / \sigma_{Q}$, indicating a picture of transport due to vortices of net density $B / \phi_{0}$. The value of $\kappa_{x x}$ has the same dependence upon all parameters as that obtained by Bhaseen et al. [12]. We can also compare the value of the numerical prefactor. For $\Phi_{\sigma}$ we use the value $1.037 / \epsilon^{2}$ obtained in Ref. 17 in the $\epsilon$-expansion $(\epsilon=3-d$ where $d$ is the spatial dimension), which is the same expansion by Bhaseen et al. [12]. It is also easy to compute the value of $\Phi_{\varepsilon}+\Phi_{P}$ in the same expansion: to the leading order needed, these are just given by the values for free, massless, relativistic bosons in $d=3$, which yields $\Phi_{\varepsilon}+\Phi_{P}=4 \pi^{2} / 45+\mathcal{O}(3-d)$. Using these values we obtain the same result for $\kappa_{x x}$ as in Eq. (24) of Ref. 12, with their dimensionless parameter $g=4.66$ (not to be confused with our coupling $g$ ). This is to be compared with their value $g=5.55$. The origin of this numerical discrepancy is not clear to us. We believe Eq. (1.28) is an exact identity in $d=2$, but it is possible that it is modified when $d$ is close to 3 .

The outline of the paper is as follows. Section II will compare the result for the Nernst effect and the thermoelectric response Eq. (1.26) with experiments on the cuprate superconductors and on $\mathrm{Nb}_{0.15} \mathrm{Si}_{0.85}$ films. Section III will present the derivation of these results using a hydrodynamic analysis of transport near a generic, $2+1$ dimensional, "relativistic" quantum critical point perturbed by a chemical potential, a magnetic field, and weak impurity scattering. An estimation of the impurity scattering rate appears in Section IV. Section V will describe the exact solution for transport near a supersymmetric quantum critical point, perturbed by a chemical potential and a magnetic field, which is solvable by the AdS/CFT mapping to the physics of a dyonic black hole in $3+1$ spacetime dimensions. Some technical details appear in the appendices.

\section{COMPARISON WITH EXPERIMENTS}

Our main results for the Nernst signal have already been stated in Section ID. In the following subsections, we will these results with recent observations in the cuprate superconductors [1-4], and also briefly discuss experiments in $\mathrm{Nb}_{0.15} \mathrm{Si}_{0.85}$ films [5, 6]. As mentioned before, in most of these experiments the electrical conductivity is dominated by non-critical fermionic contributions which are not captured by our relativistic hydrodynamic description. On the other hand, the transverse thermo-electric response $\alpha_{x y}$ is expected to be predominantly due to superconducting fluctuations and the vortex liquid. In practice $\alpha_{x y}$ is conveniently measure via the Nernst signal, using the relation $\alpha_{x y} \approx \sigma_{x x} \vartheta_{y x}$. The latter holds if the non-critical Hall conductivity is small, $\sigma_{x y} \ll \sigma_{x x}$, as is usually the case.

It is convenient to perform the experimental comparisons by rescaling $B$ and $\rho$ so that they are both measured in units of (energy $)^{2}$,

$$
B=\bar{B} \phi_{0} /(\hbar v)^{2} \quad ; \quad \rho=\bar{\rho} /(\hbar v)^{2} .
$$

Further we observe that in typical experiments the flux per (excess) particle is very small, 
$B / \rho \ll 1$ and therefore $\gamma / \omega_{c} \ll 1$. In this regime Eq. (1.26) simplifies to

$$
\begin{aligned}
\left(\frac{h}{2 e k_{B}}\right) \alpha_{x y} & \approx \frac{s / k_{B}}{B / \phi_{0}}\left(\tau_{\mathrm{imp}} \omega_{c}\right)^{2} \frac{1+\gamma /\left(\tau_{\mathrm{imp}} \omega_{c}^{2}\right)(1-\mu \rho / s T)}{1+\left(\tau_{\mathrm{imp}} \omega_{c}\right)^{2}} \\
& \approx \Phi_{s} \bar{B}\left(k_{B} T\right)^{2}\left(\frac{2 \pi \tau_{\mathrm{imp}}}{\hbar}\right)^{2} \frac{\bar{\rho}^{2}+\Phi_{\sigma} \Phi_{\varepsilon+P}\left(k_{B} T\right)^{3} \hbar / 2 \pi \tau_{\mathrm{imp}}}{\Phi_{\varepsilon+P}^{2}\left(k_{B} T\right)^{6}+\bar{B}^{2} \bar{\rho}^{2}\left(2 \pi \tau_{\mathrm{imp}} / \hbar\right)^{2}}
\end{aligned}
$$

where in the second line we have assumed a fully relativistic regime with $s \sim T^{2}$ and $\varepsilon+P \sim T^{3}$, and $\mu \rho \ll s T$. We recall that $\Phi_{\varepsilon+P}$ and $\Phi_{\sigma}$ are universal functions of $\mu / T$, and have an additional dependence on $\left(g-g_{c}\right) / T^{1 / \nu}$.

\section{A. The cuprates}

Given the relative simplicity of our model of the cuprate superconductors, detailed quantitative comparisons with the observations of Ref. 4 are probably premature. In particular, we have omitted the influence of long-range Coulomb interactions, which modifies the spectrum of boson density fluctuations, and likely leads to a superfluid-insulator quantum critical point which is not Lorentz invariant [35,36]. Also, although Dirac fermion excitations are included in some of the CFTs mentioned above (corresponding to the nodal points of the $d$-wave superconductor) other Fermi excitations associated with a Fermi surface may also be important, especially in the case of NbSi. Keeping these caveats in mind, it is nevertheless useful to examine the extent to which the present model can describe the observations. As we will now show, using physically reasonable values of the parameters in the theory, our results describe the overall absolute magnitude of the observations, and numerous qualitative trends [4] remarkably well.

We work here with a simple caricature of our predictions: We ignore the $T$ and $\rho$ dependence of the universal functions and simply treat them as constants, $\Phi_{\sigma} \approx 1.037$, $\Phi_{\varepsilon+P}^{(2 d)} \approx \Phi_{s}^{(2 d)} \approx 3 \zeta(3) / \pi \approx 1.148$. This is equivalent to assuming in Figs. 1,2 that $g=g_{c}$ and $\mu=\rho=0$ for the purpose of evaluating these functions. It is not difficult to extend our theory to include the influence of these corrections to the leading quantum-critical behavior, but such a detailed analysis would not be commensurate with the other simplifications noted above.

We notice that for small $B$, Eqs. $(2.2,2.3)$ predict a Nernst signal linear in $B$. At not too large temperatures, the second term in the numerator of (2.3) can be neglected and the ratio $\alpha_{x y} / B$ is seen to increase with decreasing temperature as $1 / T^{4}$,

$$
\frac{\alpha_{x y}}{B}(B \rightarrow 0) \approx\left(\frac{2 e k_{B}}{h \phi_{0}}\right) \frac{\Phi_{s}}{\Phi_{\varepsilon+P}^{2}}\left(\frac{2 \pi \tau_{\mathrm{imp}}}{\hbar}\right)^{2} \frac{\rho^{2}(\hbar v)^{6}}{\left(k_{B} T\right)^{4}} .
$$

Such a power law with exponent 4 was indeed observed over two orders of magnitude in signal strength in underdoped $\mathrm{La}_{2-\delta} \mathrm{Sr}_{\delta} \mathrm{CuO}_{4}$ (LSCO, $\delta \leq 0.12$ ), cf., Fig. 4 in Ref. 10. Assuming 


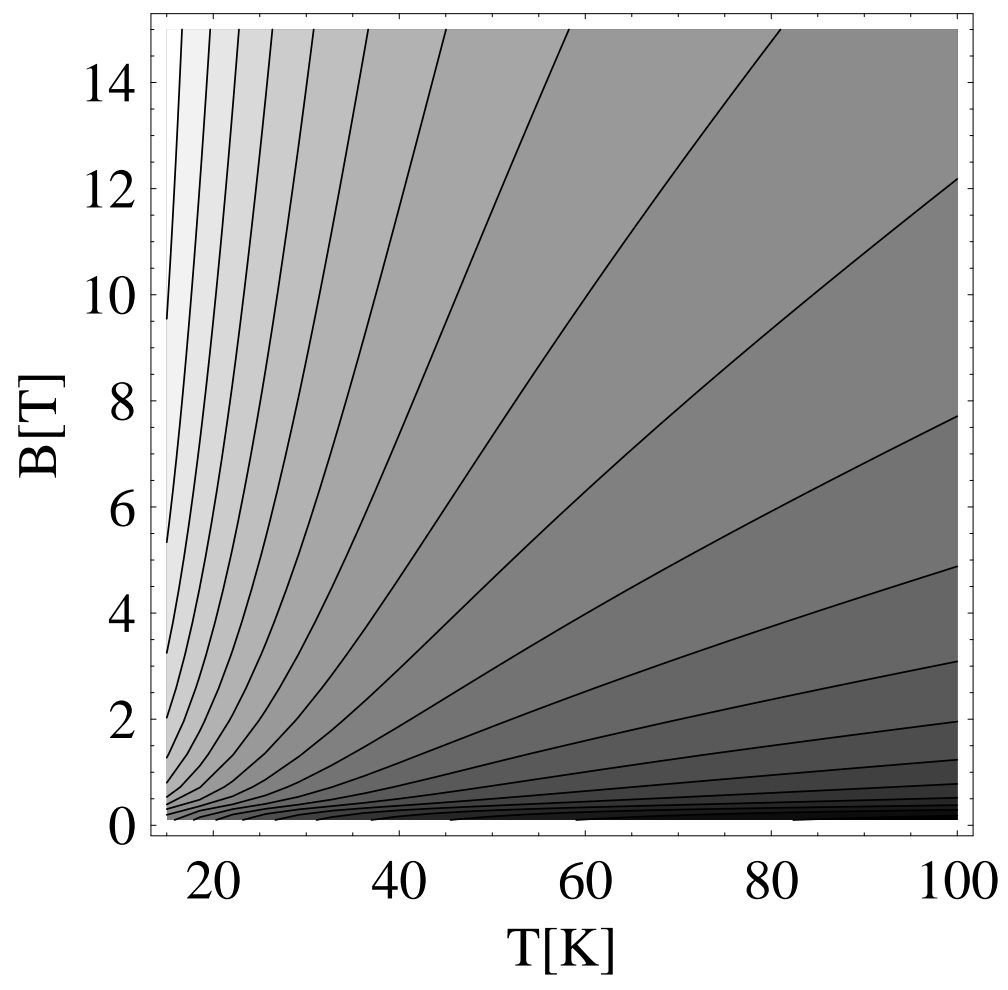

FIG. 3: Contour plot (with logarithmic spacing) of the thermoelectric conductivity $\alpha_{x y}$ (Eq. 2.3) as a function of temperature $T$ and magnetic field $B$, for parameters $\hbar v=47 \mathrm{meV} \AA, \delta-\delta_{I}=0.025$ and $\tau_{\text {imp }}=10^{-12}$ s estimated for LSCO. In the ordered low temperature regime $T<T_{c} \approx 30 \mathrm{~K}$, Eq. (2.3) will receive modifications.

a typical doping $\delta-\delta_{I}=-0.025$ for underdoped LSCO with a lattice constant $a=3.78$ $\AA$, we obtain a constraint for $\tau_{\text {imp }} v^{3}$ from fitting (2.4) to the experimental value $\alpha_{x y} / B=$ $0.48 /(T / 30 \mathrm{~K})^{4} \mathrm{nA} / \mathrm{KT}$ per layer [10]. Assuming a typical scattering time $\tau_{\text {imp }} \approx 10^{-12} \mathrm{~s}$, we obtain an estimate for the velocity $\hbar v \approx 47 \mathrm{meV} \AA$. These are reasonable parameter values, with the velocity $v$ being of the order of the characteristic velocity found in Ref. 37.

The result of Eq. (2.2) is plotted as function of both $T$ and $B$ in Fig. 3. This contour plot should be compared, e.g., with Fig. 13 in Ref. 4 in the underdoped regime, above the superconducting transition $T_{c}$.

Using the above parameter estimates we predict the cyclotron resonance

$$
\omega_{c}=6.2 \mathrm{GHz} \cdot \frac{B}{1 \mathrm{~T}}\left(\frac{35 \mathrm{~K}}{T}\right)^{3},
$$

which, at $T=35 \mathrm{~K}$, is by a factor $\omega_{c} / \omega_{c}^{(\mathrm{el})}=2 m^{(\mathrm{el})} v^{2} \cdot \rho /(\varepsilon+P) \approx 0.035$ smaller than the cyclotron frequency of free electrons. However, as mentioned before, this resonance can only be observed in ultrapure samples where $1 / \tau_{\text {imp }} \ll \omega_{c}$, which is clearly not the case in LSCO.

Having estimated the velocity $v$ and the scattering rate $\tau_{\text {imp }}$, we can make a quantitative prediction for the Nernst signal in the vicinity of a quantum critical point where the entire 


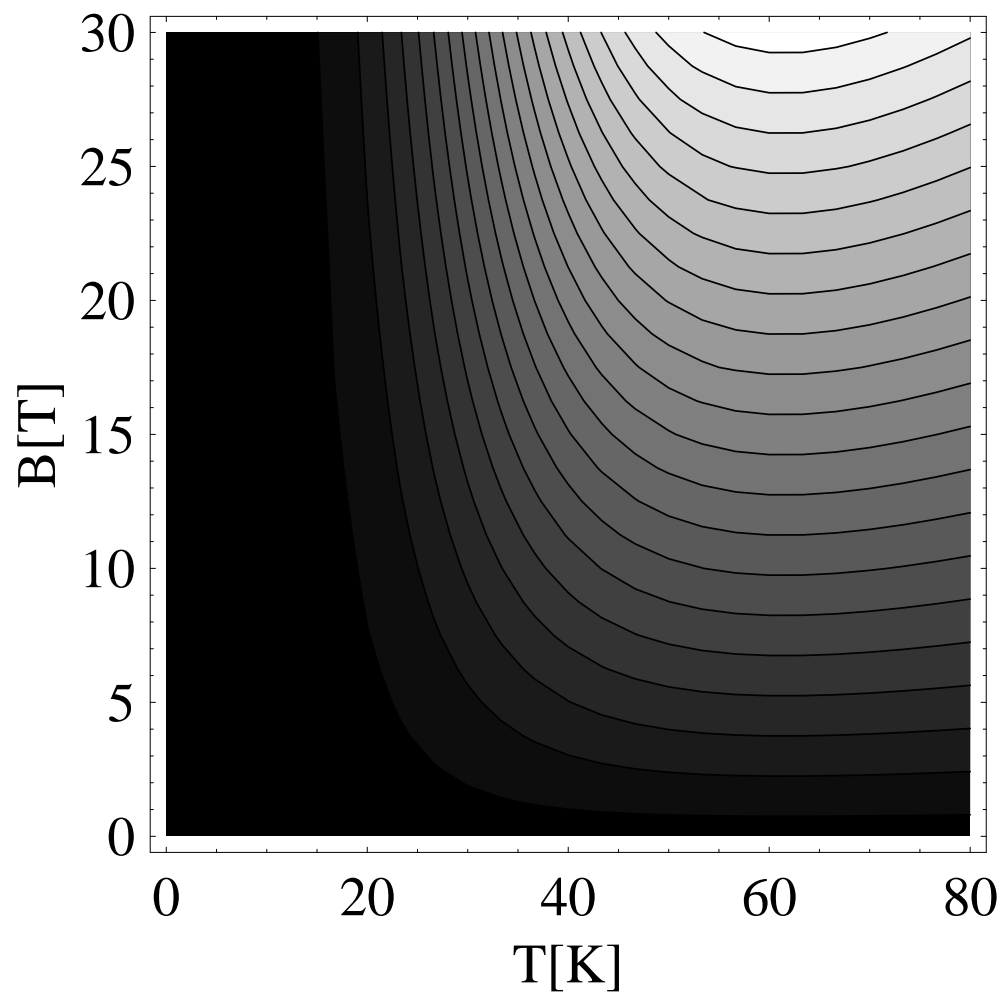

FIG. 4: Contour plot (linear scale) of the Nernst signal $e_{N}=\vartheta_{y x}$ (Eq. 2.6) close to a quantum critical point, as a function of temperature $T$ and magnetic field $B$. The parameters are the same as for Fig. 3. The signal strength in the plot ranges up to $10 \mu \mathrm{V} / \mathrm{K}$.

thermo-electric response is expected to be dominated by critical fluctuations. In this case, Eq. (1.23) can be cast into the form

$$
\vartheta_{y x}=\left(\frac{k_{B}}{2 e}\right) \frac{\left(\Phi_{\varepsilon}+\Phi_{P}\right)^{2} \Phi_{\sigma}^{2} \bar{B}\left(k_{B} T\right)^{5}\left(\hbar /\left(2 \pi \tau_{\mathrm{imp}}\right)\right)}{\left[\bar{\rho}^{2}+\Phi_{\sigma}\left(\Phi_{\varepsilon}+\Phi_{P}\right)\left(k_{B} T\right)^{3}\left(\hbar /\left(2 \pi \tau_{\mathrm{imp}}\right)\right)\right]^{2}+\Phi_{\sigma}^{2} \bar{\rho}^{2} \bar{B}^{2}},
$$

which is plotted in Fig. 4

\section{Hall resistance}

Very recently, measurements of the Hall resistance in the high field normal state of $\mathrm{YBa}_{2} \mathrm{Cu}_{3} \mathrm{O}_{6.5}$ have been reported [38]. The focus of the authors was on magnetoresistance oscillations; these oscillations are quantum interference effects which cannot be reproduced by the effective classical hydrodynamic models employed here (under the condition in Eq. (1.7)), and so are beyond the scope of the present paper. However, the authors also reported a background Hall resistance which, surprisingly, was negative. The sample has hole density $\delta=0.1$. As argued in Section I, the density of mobile carriers, $\rho$, which appears in the hydrodynamic theory [19] (and which contributes a Magnus force on vortices [20]) is given 
by the difference in density between the superconductor and the proximate Mott insulator. Using an insulator at $\delta_{I}=0.125$, we have $\rho=-0.025 /\left(2 a^{2}\right)$. This negative value of $\rho$ provides a very natural explanation of the observed negative Hall resistance. Also, we can predict that the Hall resistance should change sign as $\delta$ is increased beyond $\delta_{I}$.

We can make a more quantitative comparison with experiments. In Eq. (3.38) in Section III A, we report the value of the Hall resistivity, $\rho_{x y}$, and the zero frequency limit of that result is

$$
\rho_{x y}=\frac{B}{2 e \rho c}\left[1-\frac{\left(1 / \tau_{\text {imp }}\right)^{2}}{\left(1 / \tau_{\text {imp }}+\omega_{c}^{2} / \gamma\right)^{2}+\omega_{c}^{2}}\right] ;
$$

in the absence of impurity scattering $\left(\tau_{\mathrm{imp}} \rightarrow \infty\right)$, this result was noted in Ref. 29. Using the value of $\rho$ noted above, at $B=60 \mathrm{~T}$, we determine that the prefactor of the square brackets in Eq. (2.7) is $-2.2 \mathrm{k} \Omega$. For the factor within the square brackets, we assume the same parameters as found above for LSCO, and conclude that it is close to unity. This result is to be compared with the observed resistance per layer [38] at this field of $-3.9 \mathrm{k} \Omega$, which is quite reasonable agreement for this simple model.

\section{B. $\mathrm{NbSi}$}

We also note the experiments on amorphous films of $\mathrm{Nb}_{0.15} \mathrm{Si}_{0.85}$ reported in Refs. 5, 6 The normal phase, $T>T_{c}$, of these films exhibits a number of features that are consistent with our hydrodynamic results, when taken to their non-relativistic limit. In particular, $\alpha_{x y}$ as given in Eq. (2.2) displays a functional dependence on magnetic field akin to that reported in Ref. 6:

$$
\alpha_{x y} \propto \frac{B}{\xi^{-4}+\ell_{B}^{-4}} \propto \frac{B}{1+\left(B / B_{0}\right)^{2}},
$$

with $B_{0}=\hbar c / e \xi^{2}$, which was interpreted as the physics being controlled by the shorter of the superconducting coherence length $\xi$ and the magnetic length $\ell_{B}=(\hbar c / e B)^{1 / 2}[6]$. We mention that the low $B$ data, i.e., the Nernst coefficient $\nu=\lim _{B \rightarrow 0} \alpha_{x y} / B \sigma_{x x}$ measured in Ref. 5, was very successfully described by the theory of Gaussian fluctuations [8]. However, the crossover (2.8) and the high field behavior $\alpha_{x y} \sim 1 / B$ remained unexplained in earlier theories. Our magnetohydrodynamic approach may give a hint to the origin of the latter. We believe that the similarity of Eqs. (2.2) and (2.8) is not a mere coincidence. Rather, it leads us to speculate that the scattering time $\tau_{\text {imp }}$ should be identified with

$$
\tau_{\mathrm{imp}}=\frac{B}{B_{0} \omega_{c}}=\frac{m \xi^{2}}{\hbar} \approx\left(k_{F} \ell\right) \frac{\xi^{2}}{v_{F} \ell} \sim \tau_{\mathrm{GL}},
$$

the Ginzburg-Landau life time of fluctuating Cooper pairs [39]. Here we have used the free

electron value (non-relativistic limit) for the cyclotron frequency $\omega_{c}^{(\mathrm{el})}=e B / m^{(\mathrm{el})} c$. Further, $v_{F}=\hbar k_{F} / m$ is the Fermi velocity, and we have used that $k_{F} \ell \approx \mathcal{O}(1)$ in the studied amorphous NbSi [5]. The estimate $\tau_{\mathrm{imp}} \approx \tau_{\mathrm{GL}}$ suggests that the suppression of the Nernst 
signal at high fields is due to the Cooper pairs starting to perform entire orbits over their life time.

We may use the above guess of $\tau_{\text {imp }}$ to express the low field limit of $\alpha_{x y}$, as

$$
\alpha_{x y}\left(B \ll B_{0}\right)=\frac{k_{B} e}{\hbar} \frac{\xi^{2}}{\ell_{B}^{2}} \frac{s \xi^{2}}{k_{B}},
$$

where we have invoked a small value of $\gamma$ to approximate the last numerator in Eq. (2.2) by to 1 . It is interesting to note that apart from the last factor which describes the entropy per coherence volume, this expression has the same parameter dependence as the one derived from Gaussian fluctuations in Ref. 8.

\section{MAGNETOHYDRODYNAMICS}

The remainder of this paper will revert to natural dimensionless units with $\hbar=k_{B}=$ $2 e=v=1$, and absorb a factor of $1 / c$ in the definition of $B$.

Here we will focus on the nature of quantum critical transport in the hydrodynamic region $[14,17]$ where $\hbar \omega \ll k_{B} T$. The condition in Eq. (1.7) ensures $\hbar \omega_{c} \ll k_{B} T$, and a relativistic formulation is appropriate if also $m_{0} \lesssim k_{B} T$ is satisfied. We will use the method described by Landau and Lifshitz [46], which was recently reviewed in the context of a string theory computation [47]. These previous analyses were carried out for $B=0$ and $1 / \tau_{\text {imp }}=0$, and only considered the longitudinal electrical and thermal conductivities. Here we will show how the hydrodynamic analysis can be extended to include non-zero values of these parameters. Further, we will obtain results for the frequency dependence of the full set of transport co-efficients in Eq. (1.13). These results are consistent with the exact results obtained via AdS/CFT for a particular SCFT which are presented in Section V and Ref. 30 - the latter results however extend over a wider regime of parameters.

The fundamental ingredients of a hydrodynamic analysis are the conserved quantities and their equations of motion. Unlike in the theory of dynamics near classical, finite temperature critical points [49], here we do not need to explicitly consider the order parameter dynamics for the effective equations of motion of the low frequency theory. The key difference is that $k_{B} T / \hbar$ constitutes an intrinsic relaxation time for the order parameter fluctuations, and we are only interested in much lower frequency scales. In contrast, at a classical critical point, the relaxation time diverges. The frequency scales larger than $k_{B} T / \hbar$ cannot be addressed by the methods below, and require a full quantum treatment of the dynamics of the CFT.

The conserved quantities of interest are the electrical charge, the energy, and the momentum. For the relativistic theories under consideration, these can be assembled into the electrical current 3-vector ${ }^{2} J^{\mu}=\left(\rho, J^{x}, J^{y}\right)$, and the stress-energy tensor $T^{\mu \nu}$. We will use

\footnotetext{
${ }^{2}$ Notice that upon restoring $v$, the 3 -current reads $J^{\mu}=(\rho v, 0,0)$.
} 
standard relativistic notation with the metric tensor $g^{\mu \nu}=\operatorname{diag}(-1,1,1)$ and coordinates $x^{\mu}=(v t, x, y)$. For the moment, we will ignore the momentum relaxation due to the weak impurity potential in Eq. (1.9), and include its effects shortly below. With total momentum conserved, the equations of motion obeyed by the total electrical, momentum and energy currents are

$$
\begin{aligned}
\partial_{\mu} J^{(\mathrm{tot}) \mu} & =0 \\
\partial_{\nu} T^{(\mathrm{tot}) \mu \nu} & =F^{\mu \nu} J_{\nu}^{(\mathrm{tot})} .
\end{aligned}
$$

The first equation represents the conservation of charge and requires no further comment. The second equation describes the evolution of the stress energy tensor, and the term on the right hand side represent the effects of the external $B$ field. Here $F^{\mu \nu}$ is the applied magnetic field which takes the fixed value ${ }^{3}$

$$
F^{\mu \nu}=\left(\begin{array}{ccc}
0 & 0 & 0 \\
0 & 0 & B \\
0 & -B & 0
\end{array}\right)
$$

and the right hand side of Eq. (3.2) describes the Lorentz force exerted by this field, as discussed, e.g., in Ref. 48. In equilibrium, we have $J^{(\text {tot }) \mu}=(\rho, 0,0)$ and then the term proportional to $F^{\mu \nu}$ vanishes, as expected.

To use the equations (3.1) and (3.2), we need to relate $J^{(\text {tot }) \mu}$ and $T^{(\text {tot }) \mu \nu}$ to parameters which define the local thermodynamic equilibrium, and a three-velocity $u^{\mu}$ which represents the velocity of the system in local equilibrium with respect to the laboratory frame. As usual [46], the three-velocity $u^{\mu} \equiv d x^{\mu} / d \tau$ satisfies $u^{\mu} u_{\mu}=-1$, and $u^{\mu}=(1,0,0)$ in the equilibrium frame where there is no energy flow ${ }^{4}$. For the thermodynamic parameters we

3 More precisely, if we also allow for an electrical field in the lab frame and restore $v$, the field tensor takes the form

$$
F^{\mu \nu}=\left(\begin{array}{ccc}
0 & (c / v) E_{x} & (c / v) E_{y} \\
-(c / v) E_{x} & 0 & B \\
-(c / v) E_{y} & -B & 0
\end{array}\right)
$$

The equation of motion (3.2) is a priori valid for velocities $v^{\prime} \ll v$, where the non-relativistic limit applies. However, it is valid as a relativistically covariant equation under Lorentz transformations $\Lambda_{\nu}^{\mu}$ characterized by the limit velocity $v(n o t c)$, if it is understood that $F^{\mu \nu}$ transforms as an antisymmetric tensor, $F^{\mu \nu}=\Lambda_{\alpha}^{\mu} \Lambda_{\beta}^{\nu} F^{\alpha \beta}$ under a change of reference frame. This is exact up to negligible corrections of order $O\left([v / c]^{2}\right)$.

4 Note that the absence of an energy flow in the rest frame defines the velocity vector $u^{\mu}$ in the "dynamic" frame used throughout this paper. Alternatively, one can formulate the hydrodynamics in the kinetic frame [45] where $\bar{u}^{\mu}$ denotes the velocity associated with the electrical current via $J^{\mu}=\rho \bar{u}^{\mu}$. However, the dynamic frame is the natural frame to work with, since we assume local equilibrium. $u^{i}$ is canonically conjugate to the momentum density $T^{0 i}$, and the energy current has a natural expression in terms of $u^{\mu}$ (cf. Eq. (3.18)). 
will use the charge density, $\rho$, the energy density $\varepsilon$, the pressure $P$, and the magnetization density $M$; we define the pressure, $P$, as the negative of the grand potential per unit volume, and $M$ as the derivative of the latter with respect to $B$.

Using these parameters the stress energy tensor of a fluid is given by

$$
\begin{aligned}
T^{(t o t) \mu \nu} & =T^{\mu \nu}-M^{\mu \gamma} F_{\gamma}^{\nu}+T^{E \mu \nu}, \\
T^{\mu \nu} & =(\varepsilon+P) u^{\mu} u^{\nu}+P g^{\mu \nu}+\tau^{\mu \nu},
\end{aligned}
$$

where

$$
M^{\mu \nu}=\left(\begin{array}{ccc}
0 & 0 & 0 \\
0 & 0 & M \\
0 & -M & 0
\end{array}\right),
$$

is the polarization tensor [44]. (The electric polarizations $M^{0 i}=-M^{i 0}$ vanish in the absence of electric fields in the lab frame.)

The electrical current is given by

$$
\begin{aligned}
J^{(\text {tot }) \mu} & =J^{\mu}+\partial_{\nu} M^{\mu \nu}, \\
J^{\mu} & =\rho u^{\mu}+\nu^{\mu} .
\end{aligned}
$$

The 'dissipative current' $\nu^{\mu}$ accounts for the fact that the charge current and the energy current are not simply proportional to each other. This is because there is a heat flow even in the absence of matter convection, which is a consequence of particle-antiparticle creation and annihilation.

We have introduced the transport currents [43], $J^{\mu}$ and $T^{\mu \nu}$ which represent observable quantities which can couple to probes external to the system. The remaining contribution to $J^{(\text {tot }) \mu}$ is the magnetization current $[8,10,42,43]$, which is induced due to spatial variations in the local magnetization density. The coupling of the magnetization to the magnetic field contributes an extra contribution $-M^{\mu \gamma} F_{\gamma}^{\nu}$ to the stress energy tensor, reducing its spatial diagonal to $P_{\text {int }}=P-M B$ (see also Appendix B). The origin and the physics of this term has also been discussed by Cooper et al [43]. Finally $T^{E \mu \nu}$ represents the "energy magnetization current". We will not need an explicit expression for this quantity here, apart from the fact that it obeys $\partial_{\nu} T^{E \mu \nu}=0$. Expressions will be given later in the paper when we consider specific CFTs: for the super Yang Mills theory in Section V, and for the Wilson-Fisher fixed point in Appendix B. With these magnetization currents subtracted out, the residual transport currents continue to obey the equations of motion as in Eqs. (3.1) and (3.2):

$$
\begin{aligned}
\partial_{\mu} J^{\mu} & =0, \\
\partial_{\nu} T^{\mu \nu} & =F^{\mu \nu} J_{\nu} .
\end{aligned}
$$

In the expressions for the transport currents in Eqs. (3.5) and (3.8), we assume that $\varepsilon, P$, $\rho$, and $M$ are thermodynamic functions of the local chemical potential, $\mu$, the temperature 
$T$, and the magnetic field $B$. We will treat $u^{\mu}, \mu$, and $T$ as the "independent" degrees of freedom which respond to external perturbations, and assume that the remaining thermodynamic variables will follow according to the equation of state. In equilibrium, the non-zero components of $J^{\mu}$ and $T^{\mu \nu}$ are

$$
J^{t}=\rho ; \quad T^{t t}=\varepsilon \quad ; \quad T^{x x}=T^{y y}=P-M B .
$$

Eqs. (3.5) and (3.8) also contain the dissipative components of the stress-energy tensor and the current, as introduced in Ref. 46; these are are orthogonal to $u^{\mu}$

$$
u_{\mu} \tau^{\mu \nu}=u_{\mu} \nu^{\mu}=0,
$$

and will be determined below by imposing the requirement that the total entropy increases under time evolution.

We are now in a position to introduce the scattering due to a dilute concentration of impurities. We assume that their dominant effect is to introduce a relaxation in the local transport momentum density: impurity scattering conserves charge and energy, and we do not expect the magnetization currents to relax by impurity scattering. Thus, we modify Eqs. (3.9) to

$$
\begin{aligned}
\partial_{\mu} J^{\mu} & =0, \\
\partial_{\nu} T^{\mu \nu} & =F^{\mu \nu} J_{\nu}+\frac{1}{\tau_{\mathrm{imp}}}\left(\delta_{\nu}^{\mu}+u^{\mu} u_{\nu}\right) T^{\nu \gamma} u_{\gamma} .
\end{aligned}
$$

The new term in the second equation in Eq. (3.12) represents the impurity scattering. The impurities, as described by the random potential in Eq. (1.9) are assumed to be at rest in the laboratory frame. The projection operators built out of the $u^{\mu}$ in the second term in Eq. (3.2) ensure that in the laboratory frame only the total momentum, i.e., $T^{i 0}$, is relaxed. We will discuss a computation of the value of $\tau_{\text {imp }}$ later in Section IV.

Following Landau and Lifshitz [46], we now use the positivity of the entropy production to constrain the expression for the dissipative components $\nu^{\mu}$ and $\tau^{\mu \nu}$. First we notice from Eq. (3.5) that

$$
u_{\nu} \partial_{\mu} T^{\mu \nu}=-(\varepsilon+P) \partial_{\mu} u^{\mu}-u^{\mu} \partial_{\mu} \varepsilon+u_{\nu} \partial_{\mu} \tau^{\mu \nu},
$$

and from Eq. (3.12) that

$$
u_{\nu} \partial_{\mu} T^{\mu \nu}=F^{\mu \nu} u_{\mu} \nu_{\nu}
$$

Using the thermodynamic relations

$$
\varepsilon+P=T s+\mu \rho, \quad d \varepsilon=T d s+\mu d \rho,
$$

Eq. (3.11) and current conservation, Eqs. (3.13) and (3.14) can be transformed into

$$
\partial_{\mu}\left(s u^{\mu}\right)=\frac{\mu}{T} \partial_{\mu} \nu^{\mu}-\frac{1}{T} F^{\mu \nu} u_{\mu} \nu_{\nu}-\frac{\tau^{\mu \nu}}{T} \partial_{\mu} u_{\nu},
$$


or

$$
\partial_{\mu}\left(s u^{\mu}-\frac{\mu}{T} \nu^{\mu}\right)=\nu^{\mu}\left[\frac{1}{T}\left(-\partial_{\mu} \mu+F^{\mu \nu} u_{\nu}\right)+\mu \frac{\partial_{\mu} T}{T^{2}}\right]-\frac{\tau^{\mu \nu}}{T} \partial_{\mu} u_{\nu} .
$$

It is natural to interpret the left hand side as the divergence of the entropy current. Accordingly we can interpret the three vector

$$
Q^{\mu}=s T u_{d}^{\mu}-\mu \nu^{\mu}=(\varepsilon+P) u^{\mu}-\mu J^{\mu} \equiv J^{E \mu}-\mu J^{\mu}
$$

as the heat current. We have also introduced the energy current $J^{E \mu}=(\varepsilon+P) u^{\mu}$.

Since the entropy can only increase, the right hand side of (3.17) must be positive. Generalizing the arguments of Landau and Lifshitz, we deduce the most general expressions for the dissipative currents which are linear in spatial gradients and the velocity,

$$
\begin{aligned}
\nu^{\mu} & =\sigma_{Q}\left(g^{\mu \nu}+u^{\mu} u^{\nu}\right)\left[\left(-\partial_{\nu} \mu+F_{\nu \lambda} u^{\lambda}\right)+\mu \frac{\partial_{\mu} T}{T}\right], \\
\tau^{\mu \nu} & =-\left(g^{\mu \lambda}+u^{\mu} u^{\lambda}\right)\left[\eta\left(\partial_{\lambda} u^{\nu}+\partial^{\nu} u_{\lambda}\right)+(\zeta-\eta) \delta_{\lambda}^{\nu} \partial_{\alpha} u^{\alpha}\right] .
\end{aligned}
$$

Here $\eta$ and $\zeta$ are the shear and bulk viscosities, and $\sigma_{Q}$ is a conductivity. Notice that there are only three independent transport co-efficients. We will neglect velocity gradients for the most part in this paper, and so the viscosities do not appear in our main results. Consequently, we have the remarkable feature that all transport response functions depend only upon a single dissipative transport co-efficient $\sigma_{Q}$. Notice that in the dissipative current the gradient of the chemical potential appears in combination with the electromagnetic forces $F^{\mu \nu} u_{\nu}$, which is natural since it is equivalent to an electric field.

\section{A. Linear response}

We will now follow the strategy of Kadanoff and Martin [50]: Use the equations of hydrodynamics to solve the initial value problem in linear response, and compare the results to those obtained from the Kubo formula in order to extract transport coefficients and their frequency dependence.

First, we address the solution of the initial value problem in hydrodynamics. We begin by choosing our independent variables: from the structure of the above equations, it appears convenient to choose the four variables $T, \mu$, and $u^{x}$ and $u^{y}$. So we write

$$
\begin{aligned}
& \mu(r, t)=\mu+\delta \mu(r, t), \\
& T(r, t)=T+\delta T(r, t),
\end{aligned}
$$

while $F^{\mu \nu}$ is fixed at the value in Eq. (3.4). We also write $u^{\mu}$ as

$$
u^{\mu}=\left(\begin{array}{c}
1 \\
v_{x}(r, t) \\
v_{y}(r, t)
\end{array}\right),
$$


where $v_{x}, v_{y}$ are of the same order as $\delta \mu$ and $\delta T$.

The other variables, $\varepsilon, P$, and $\rho$ are constrained by local thermodynamic equilibrium to have the form

$$
\begin{gathered}
\rho(r, t)=\rho+\delta \rho \equiv \rho+\left.\frac{\partial \rho}{\partial \mu}\right|_{T} \delta \mu+\left.\frac{\partial \rho}{\partial T}\right|_{\mu} \delta T, \\
\varepsilon(r, t)=\varepsilon+\delta \varepsilon \equiv \varepsilon+\left.\frac{\partial \varepsilon}{\partial \mu}\right|_{T} \delta \mu+\left.\frac{\partial \varepsilon}{\partial T}\right|_{\mu} \delta T, \\
P(r, t)=P+\delta P \equiv P+\rho \delta \mu+s \delta T .
\end{gathered}
$$

The various components of the stress-energy tensor and the current vector are perturbed accordingly. To linear order we have

$$
\begin{aligned}
\delta T^{t t} & =\delta \varepsilon \\
\delta T^{t i} & =T^{t i}=(\varepsilon+P) v_{i} \\
\delta T^{i j} & =\delta P \delta_{i j}-\eta\left(\partial_{i} v_{j}+\partial_{j} v_{i}-\delta_{i j} \partial_{k} v_{k}\right)-\zeta \delta_{i j} \partial_{k} v_{k} \\
\delta J^{t} & =\delta \rho \\
\delta \vec{J} & =\vec{J}=\rho \vec{v}+\vec{\nu} \\
\vec{\nu} & =\sigma_{Q}\left(-\vec{\nabla} \mu+\vec{v} \times \vec{B}+\mu \frac{\vec{\nabla} T}{T}\right) .
\end{aligned}
$$

For small perturbations the conservation laws take the form

$$
\begin{aligned}
\partial_{t} \rho+\vec{\nabla} \vec{J} & =0, \\
\partial_{t} \varepsilon+\vec{\nabla} \vec{J}^{E} & =0, \\
\partial_{t} \vec{J}^{E} & =-\vec{\nabla} P-\eta \vec{\nabla}^{2} \vec{v}-\zeta \vec{\nabla}(\vec{\nabla} \cdot \vec{v})+\vec{J} \times \vec{B},
\end{aligned}
$$

with the energy and heat currents

$$
\begin{aligned}
\vec{J}^{E} & =(\varepsilon+P) \vec{v} \\
\vec{Q} & =\vec{J}^{E}-\mu \vec{J}=(\varepsilon+P) \vec{v}-\rho \mu \vec{v}-\mu \vec{\nu}=s T \vec{v}-\mu \vec{\nu}
\end{aligned}
$$

The crucial remnant of the relativistic theory in the linearized hydrodynamics is the fact that the energy and particle currents are in general not parallel,

$$
\begin{aligned}
\vec{J}^{E} & =\frac{\varepsilon+P}{\rho}\left[\vec{J}-\sigma_{Q}\left(-\vec{\nabla} \mu+\vec{v} \times \vec{B}+\mu \frac{\vec{\nabla} T}{T}\right)\right] \\
& =\frac{\varepsilon+P}{\rho} \vec{J}+\frac{(\varepsilon+P)^{2}}{T \rho^{2}} \sigma_{Q} \vec{\nabla} T+\frac{\varepsilon+P}{\rho^{2}} \sigma_{Q}(-\vec{\nabla} P+\vec{J} \times \vec{B}),
\end{aligned}
$$

where we have used (3.15) to rewrite the dissipative current. The energy current consists of three parts: the first two are familiar from non-relativistic theory as the convection of 
matter and heat flow due to a thermal gradient, with a thermal conductivity [46] $\bar{\kappa}=$ $\sigma_{Q}(\varepsilon+P)^{2} /\left(T \rho^{2}\right)$. The last term in (3.30) is proportional to the acceleration vector, and is a purely relativistic phenomenon [51]. One can easily see that this term is responsible for the damping $\gamma$ of the cyclotron mode (cf., Eq. (1.19)), by using the above relation to substitute for $\vec{J}$ in the momentum conservation law (3.27).

To complete the hydrodynamic analysis, we solve the equations (3.25)-(3.27) for arbitrary initial values $\delta T^{0}, \delta \mu^{0}$ and $v_{x}^{0}=v_{y}^{0}=0$, and obtain the response in the electrical current $\vec{J}$ and the heat current $\vec{Q}$. The 'heat density' associated to the latter, $q(r)=\varepsilon(r)-\mu \rho(r)$ is canonically conjugate to the temperature at fixed chemical potential [50].

After a Fourier transform in space and a Laplace transform in time, the linear response of any quantity $A$ obeys

$$
\begin{gathered}
A(\vec{k}, \omega)=\frac{G_{A ; \varepsilon-\mu \rho}(\vec{k}, \omega)-G_{A ; \varepsilon-\mu \rho}(\vec{k}, 0)}{i \omega} \frac{\delta T^{0}(\vec{k})}{T}+\frac{G_{A ; \rho}(\vec{k}, \omega)-G_{A ; \rho}(\vec{k}, 0)}{i \omega} \delta \mu^{0}(\vec{k}) \\
+\frac{G_{A ; T^{0 i}}(\vec{k}, \omega)-G_{A ; T^{0 i}}(\vec{k}, 0)}{i \omega} \sum_{i=x, y} v^{i}(\vec{k}),
\end{gathered}
$$

where the coefficients are related to retarded equilibrium correlation functions, as can be shown from analyzing an adiabatic perturbation [50] of the form

$$
\delta \mathcal{H}(t)=-\int d r\left[\delta \mu(r, t) n(r, t)-\frac{\delta T(r, t)}{T}(\varepsilon(r, t)-\mu n(r, t))-\sum_{i} v^{i}(r, t) T^{0 i}(r, t)\right]
$$

Finally, using the conservation laws in the form

$$
\begin{aligned}
i \omega \rho(\vec{k}) & =i \vec{k} \vec{J}(\vec{k}), \\
i \omega(\varepsilon(\vec{k})-\mu \rho(\vec{k})) & =i \vec{k}\left[\vec{J}^{E}(\vec{k})-\mu \vec{J}(\vec{k})\right]=i \vec{k} \vec{Q}(\vec{k}),
\end{aligned}
$$

we obtain

$$
\begin{gathered}
A(\vec{k}, \omega)=-\frac{1}{i \omega}\left[\frac{G_{A ; \vec{Q}}(\vec{k}, \omega)-G_{A ; \vec{Q}}(\vec{k}, 0)}{i \omega}\left(-\frac{\vec{\nabla} T^{0}(\vec{k})}{T}\right)+\frac{G_{A ; \vec{J}}(\vec{k}, \omega)-G_{A ; \vec{J}}(\vec{k}, 0)}{i \omega} \vec{E}(\vec{k})\right] \\
+\frac{G_{A ; T^{0 i}}(\vec{k}, \omega)-G_{A ; T^{0 i}}(\vec{k}, 0)}{i \omega} \sum_{i=x, y} v^{i}(\vec{k}) .
\end{gathered}
$$

For $A=\vec{J}$ and $A=\vec{Q}$ one recognizes the co-efficients of $\vec{E} \equiv-\vec{\nabla} \mu^{0}$ and $\left(-\vec{\nabla} T^{0} / T\right)$ as $(-1 / i \omega)$ times the Kubo formulae for the thermoelectric co-efficients $\hat{\sigma}, \hat{\alpha}, \hat{\kappa}$. The response to an initial velocity perturbation could be used to extract frequency dependent viscous response functions.

After a Laplace transform in time, the Eqs. (3.25) and (3.26) take the form

$$
\begin{aligned}
i\left(\left.\frac{\partial \rho}{\partial \mu}\right|_{T} \delta \mu^{0}+\left.\frac{\partial \rho}{\partial T}\right|_{\mu} \delta T^{0}\right) & =\omega\left(\left.\frac{\partial \rho}{\partial \mu}\right|_{T} \delta \mu+\left.\frac{\partial \rho}{\partial T}\right|_{\mu} \delta T\right)+i \vec{\nabla}\left(\rho \vec{v}+B \sigma_{Q} \hat{\epsilon} \vec{v}\right)-i \sigma_{Q} T \nabla^{2}\left(\frac{\mu}{T}\right), \\
i\left(\left.\frac{\partial \varepsilon}{\partial \mu}\right|_{T} \delta \mu^{0}+\left.\frac{\partial \varepsilon}{\partial T}\right|_{\mu} \delta T^{0}\right) & =\omega\left(\left.\frac{\partial \varepsilon}{\partial \mu}\right|_{T} \delta \mu+\left.\frac{\partial \varepsilon}{\partial T}\right|_{\mu} \delta T\right)+i(\varepsilon+P) \vec{\nabla} \cdot \vec{v},
\end{aligned}
$$


for charge and energy conservation, and Eq. (3.27)

$$
\begin{aligned}
i(\varepsilon+P) \vec{v}^{0}=(\omega+ & \left.i / \tau_{\text {imp }}\right)(\varepsilon+P) \vec{v}+i(\rho \vec{\nabla} \mu+s \vec{\nabla} T) \\
& -i B \rho \hat{\epsilon} \vec{v}+i B \sigma_{Q} \hat{\epsilon}\left(\vec{\nabla} \mu-\frac{\mu}{T} \vec{\nabla} T\right)+i B^{2} \sigma_{Q} \vec{v} \\
& +i \eta \nabla^{2} \vec{v}+i \zeta \vec{\nabla}(\vec{\nabla} \cdot \vec{v})
\end{aligned}
$$

for momentum conservation.

In the case of weak enough momentum relaxation, the response functions will exhibit peaks associated with the normal modes of these linearized equations. Apart from the damped cyclotron mode discussed above, one finds two diffusive modes, as analyzed in Appendix A. However, those will not be of importance below since we are restricting to long wavelengths in the sequel.

In the limit $k \rightarrow 0$, the current and energy conservation impose $\delta \mu=i \delta \mu^{0} / \omega$ and $\delta T=i \delta T^{0} / \omega$, expressing that the decay of initial perturbations can be neglected. Further, the contributions from viscosity can be neglected in this limit. Upon injection into the momentum conservation equations (3.36), we easily obtain the retarded Greens functions and via the mapping (3.34) and Kubo formulae the transport co-efficients defined in Eq. (1.13):

$$
\begin{aligned}
& \sigma_{x x}=\sigma_{Q} \frac{\left(\omega+i / \tau_{\mathrm{imp}}\right)\left(\omega+i \gamma+i / \tau_{\mathrm{imp}}+i \omega_{c}^{2} / \gamma\right)}{\left[\left(\omega+i \gamma+i / \tau_{\mathrm{imp}}\right)^{2}-\omega_{c}^{2}\right]}, \\
& \sigma_{x y}=-\frac{\rho}{B} \frac{\left(\gamma^{2}+\omega_{c}^{2}-2 i \gamma \omega+2 \gamma / \tau_{\text {imp }}\right)}{\left[\left(\omega+i \gamma+i / \tau_{\text {imp }}\right)^{2}-\omega_{c}^{2}\right]} \text {, } \\
& \alpha_{x x}=\frac{s}{B} \frac{\left[\omega_{c}\left(i \omega-1 / \tau_{\mathrm{imp}}\right)\left(1-\left(\gamma \mu \rho /\left(\omega_{c}^{2} T s\right)\right)\left(\gamma+1 / \tau_{\mathrm{imp}}-i \omega\right)\right)\right]}{\left[\left(\omega+i \gamma+i / \tau_{\mathrm{imp}}\right)^{2}-\omega_{c}^{2}\right]}, \\
& \alpha_{x y}=-\frac{s}{B} \frac{\left[\gamma^{2}+\omega_{c}^{2}+\gamma\left(-i \omega+1 / \tau_{\mathrm{imp}}\right)(1-\mu \rho /(T s))\right]}{\left[\left(\omega+i \gamma+i / \tau_{\mathrm{imp}}\right)^{2}-\omega_{c}^{2}\right]}, \\
& \bar{\kappa}_{x x}=-\left(\frac{(\varepsilon+P)^{2}}{T B \rho}\right) \frac{\omega_{c} \gamma}{\left[\left(\omega+i \gamma+i / \tau_{\text {imp }}\right)^{2}-\omega_{c}^{2}\right]} \\
& \times\left\{1+\left(1 / \tau_{\text {imp }}-i \omega\right) \frac{\left(s^{2} T^{2} \omega_{c}^{2}+\gamma^{2} \mu^{2} \rho^{2}\right)}{\gamma \omega_{c}^{2}(\varepsilon+P)^{2}}+\left(1 / \tau_{\text {imp }}-i \omega\right)^{2} \frac{\mu^{2} \rho^{2}}{\omega_{c}^{2}(\varepsilon+P)^{2}}\right\}, \\
& \bar{\kappa}_{x y}=-\left(\frac{T s^{2}}{B \rho}\right) \frac{\omega_{c}^{2}}{\left[\left(\omega+i \gamma+i / \tau_{\mathrm{imp}}\right)^{2}-\omega_{c}^{2}\right]} \\
& \times\left\{1-\frac{2 \mu \sigma_{Q} B}{T s} \frac{\left(\gamma+1 / \tau_{\text {imp }}-i \omega\right)}{\omega_{c}}-\left(\frac{\mu \sigma_{Q} B}{T s}\right)^{2}\right\} .
\end{aligned}
$$

We also computed the thermoelectric co-efficients $\alpha_{x x}, \alpha_{x y}$ by examining the heat current induced by an applied electric field, and precisely the same result as above was obtained. This confirms Onsager reciprocity which has to hold since the densities associated with the electric and heat currents are conjugate to $\delta \mu$ and $\delta T / T$, respectively. The validity of Onsager reciprocity is a strong check of the consistency of our hydrodynamic description. 
From expressions in Eq. (3.37) we can also derive the resistivities $\hat{\rho}=\hat{\sigma}^{-1}$, the Nernst responses defined in Eq. (1.16) and the thermal conductivities defined in Eq. (1.15).

$$
\begin{aligned}
\rho_{x x} & =\frac{1}{\sigma_{Q}} \frac{\left(\omega+i / \tau_{\mathrm{imp}}\right)\left(\omega+i \gamma+i / \tau_{\mathrm{imp}}+i \omega_{c}^{2} / \gamma\right)}{\left[\left(\omega+i \omega_{c}^{2} / \gamma+i / \tau_{\mathrm{imp}}\right)^{2}-\omega_{c}^{2}\right]}, \\
\rho_{x y} & =-\frac{B}{\rho} \frac{\left(\left(\omega_{c}^{2} / \gamma\right)^{2}+\omega_{c}^{2}-2 i\left(\omega_{c}^{2} / \gamma\right) \omega+2\left(\omega_{c}^{2} / \gamma\right) / \tau_{\mathrm{imp}}\right)}{\left[\left(\omega+i \omega_{c}^{2} / \gamma+i / \tau_{\mathrm{imp}}\right)^{2}-\omega_{c}^{2}\right]}, \\
\vartheta_{x x} & =\frac{s}{\rho} \frac{\left[\left(\omega_{c}^{2} / \gamma\right)^{2}+\omega_{c}^{2}+\left(\omega_{c}^{2} / \gamma\right)\left(-i \omega+1 / \tau_{\mathrm{imp}}\right)(1-\mu \rho /(T s))-\left(\mu \rho /(T s)\left(-i \omega+1 / \tau_{\mathrm{imp}}\right)^{2}\right]\right.}{\left[\left(\omega+i \gamma+i / \tau_{\mathrm{imp}}\right)^{2}-\omega_{c}^{2}\right]}, \\
\vartheta_{x y} & =-\frac{B}{T} \frac{\left(i \omega-1 / \tau_{\mathrm{imp}}\right)}{\left[\left(\omega+i \omega_{c}^{2} / \gamma+i / \tau_{\mathrm{imp}}\right)^{2}-\omega_{c}^{2}\right]}, \\
\kappa_{x x}= & \frac{(\varepsilon+P)}{T} \frac{\left(i \omega-1 / \tau_{\mathrm{imp}}-\omega_{c}^{2} / \gamma\right)}{\left[\left(\omega+i \omega_{c}^{2} / \gamma+i / \tau_{\mathrm{imp}}\right)^{2}-\omega_{c}^{2}\right]}, \\
\kappa_{x y}= & \frac{(\varepsilon+P)}{T} \frac{\omega_{c}}{\left[\left(\omega+i \omega_{c}^{2} / \gamma+i / \tau_{\mathrm{imp}}\right)^{2}-\omega_{c}^{2}\right]} .
\end{aligned}
$$

These expressions contain the main results that were quoted in Section I. Although they appear rather complicated, most of the structure is tightly constrained and the predicted dependencies on $\omega$ are robust consequences of hydrodynamics.

Significant simplifications do appear if in addition to the small $B$ assumption in Eq. (1.7), we also assume that $\rho$ is small. In particular, let us take $B \ll T^{2}, \rho \ll T^{2}$ and $\rho \sim B \sim$ $T^{3 / 2} \sqrt{\omega}$. Note that in this limit we may simplify Eq. (3.15) to $T s=\varepsilon+P$. Then, the results in Eqs. (3.37) and (3.38) take the following more compact form (we have set $1 / \tau_{\text {imp }}=0$ because, as noted in Section I, the dependence on impurity scattering is easily restored below by $\left.\omega \rightarrow \omega+i / \tau_{\text {imp }}\right)$ :

$$
\begin{aligned}
\sigma_{x x} & =\sigma_{Q} \frac{\omega\left(\omega+i \gamma+i \omega_{c}^{2} / \gamma\right)}{\left[(\omega+i \gamma)^{2}-\omega_{c}^{2}\right]} & \sigma_{x y} & =-\frac{\rho}{B} \frac{\left(\gamma^{2}+\omega_{c}^{2}-2 i \gamma \omega\right)}{\left[(\omega+i \gamma)^{2}-\omega_{c}^{2}\right]}, \\
\rho_{x x} & =\frac{1}{\sigma_{Q}} \frac{\omega\left(\omega+i \omega_{c}^{2} / \gamma+i \gamma\right)}{\left[\left(\omega+i \omega_{c}^{2} / \gamma\right)^{2}-\omega_{c}^{2}\right]} & \rho_{x y} & =\frac{B}{\rho} \frac{\left(\left(\omega_{c}^{2} / \gamma\right)^{2}+\omega_{c}^{2}-2 i\left(\omega_{c}^{2} / \gamma\right) \gamma \omega\right)}{\left[\left(\omega+i \omega_{c}^{2} / \gamma\right)^{2}-\omega_{c}^{2}\right]} \\
\alpha_{x x} & =\frac{\rho}{T} \frac{i \omega}{\left[(\omega+i \gamma)^{2}-\omega_{c}^{2}\right]}, & \alpha_{x y} & =-\frac{s}{B} \frac{\gamma^{2}+\omega_{c}^{2}-i \gamma \omega}{\left[(\omega+i \gamma)^{2}-\omega_{c}^{2}\right]} \\
\vartheta_{x x} & =\frac{s}{\rho} \frac{\left(\omega_{c}^{2} / \gamma\right)^{2}+\omega_{c}^{2}-i\left(\omega_{c}^{2} / \gamma\right) \omega}{\left[\left(\omega+i \omega_{c}^{2} / \gamma\right)^{2}-\omega_{c}^{2}\right]} & \vartheta_{x y} & =-\frac{B}{T} \frac{i \omega}{\left[\left(\omega+i \omega_{c}^{2} / \gamma\right)^{2}-\omega_{c}^{2}\right]} \\
\bar{\kappa}_{x x} & =s \frac{i \omega-\gamma}{\left[(\omega+i \gamma)^{2}-\omega_{c}^{2}\right]}, & \bar{\kappa}_{x y} & =-s \frac{\omega_{c}}{\left[(\omega+i \gamma)^{2}-\omega_{c}^{2}\right]} \\
\kappa_{x x} & =s \frac{i \omega-\omega_{c}^{2} / \gamma}{\left[\left(\omega+i \omega_{c}^{2} / \gamma\right)^{2}-\omega_{c}^{2}\right]} & \kappa_{x y} & =s \frac{\omega_{c}}{\left[\left(\omega+i \omega_{c}^{2} / \gamma\right)^{2}-\omega_{c}^{2}\right]}
\end{aligned}
$$

The above expressions are now easily observed to obey a remarkable 'self-duality' symmetry. Under the interchanges

$$
\rho \leftrightarrow B \text { and } \sigma_{Q} \leftrightarrow 1 / \sigma_{Q}
$$


the cyclotron frequency $\omega_{c}$ in Eq. (1.18) remains invariant, while the damping frequencies in Eqs. $(1.19,1.22)$ interchange

$$
\gamma \leftrightarrow \gamma_{v}=\omega_{c}^{2} / \gamma
$$

then note that the expressions for the transport co-efficients interchange as follows:

$$
\begin{gathered}
\sigma_{x x}, \sigma_{x y}, \alpha_{x x}, \alpha_{x y}, \bar{\kappa}_{x x}, \bar{\kappa}_{x y} \\
\uparrow \\
\downarrow \\
\rho_{x x},-\rho_{x y},-\vartheta_{x y},-\vartheta_{x x}, \kappa_{x x},-\kappa_{x y} .
\end{gathered}
$$

These relations are consequences of the particle-vortex duality discussed in Ref. 14, and the mapping of the transport co-efficients in Eq. (3.42) can be deduced from the mapping $E_{i} \leftrightarrow \epsilon_{i j} J_{j}$ in Eq. (1.13). These duality relations will be discussed further in the context of SCFTs solvable by AdS/CFT in Section V and in Ref. 30: in this case the duality relations will be found to hold exactly for all $\rho$ and $B$.

\section{ESTIMATING THE MOMENTUM RELAXATION RATE}

As discussed in Section I, we assume that momentum relaxation is caused by an external perturbation of the form

$$
\mathcal{S}_{\text {imp }}=\int d \tau \int d^{d} x V(x) \mathcal{O}(x, \tau),
$$

where $V(x)$ is an external potential which is random function of spatial position $x$, but independent of $\tau$, with the averages in Eq. (1.10). The operator $\mathcal{O}$ is the "thermal operator" of the CFT, i.e., the most relevant perturbation which drives the CFT massive (despite the name, it has nothing to do with temperature in the present context).

We are interested in the modification of the equation of motion of the momentum density, $T^{i 0}$ by the impurity, because this is the only quantity whose conservation law is spoiled by the presence of impurities. In the absence of other perturbations from equilibrium we observe from Eq. (3.12) that the momentum density obeys

$$
\frac{\partial T^{i t}}{\partial t}=-\frac{1}{\tau_{\mathrm{imp}}} T^{i t}+\ldots,
$$

where the ellipses indicates terms which have an explicit spatial gradient and so their spatial integral vanishes. We will describe here an estimate of $\tau_{\text {imp }}$ to order $V_{\text {imp }}^{2}$.

For definiteness, consider the Wilson-Fisher fixed point of a complex scalar $\psi$ in Eq. (1.3), although the argument easily generalizes to other CFTs. We will also ignore the influence of $B$ and $\mu$ as these are secondary perturbations. Then $\mathcal{O}=|\psi|^{2}$ and

$$
T^{i t}=\varpi^{*} \partial_{i} \psi+\text { c.c. }
$$


where $\varpi$ is canonical momentum conjugate to $\psi$. For the following, we need the commutator

$$
\Upsilon^{j}=i\left[T^{j t}, \mathcal{O}\right]=\partial_{j}\left(|\psi|^{2}\right) .
$$

We now compute $1 / \tau_{\text {imp }}$ using the memory function method $[52,53]$. From this approach, the estimate of the momentum relaxation rate is

$$
\frac{1}{\tau_{\mathrm{imp}}}=\frac{V_{\mathrm{imp}}^{2}}{\chi_{T}} \lim _{\omega \rightarrow 0} \frac{1}{\omega} \int \frac{d^{d} k}{(2 \pi)^{d}} \operatorname{Im}\left\langle\Upsilon^{i}(-k,-\omega) \Upsilon^{i}(k, \omega)\right\rangle_{\mathrm{ret}},
$$

where we are working in general $d$ dimensions, and $\chi_{T}$ is the momentum density susceptibility i.e.

$$
\chi_{T}=\int d^{d} x d \tau\left\langle T^{i t}(x, \tau) T^{i t}(0,0)\right\rangle .
$$

Using the scaling dimensions

$$
\begin{aligned}
\operatorname{dim}\left[T^{i 0}\right] & =d+1, \\
\operatorname{dim}\left[|\psi|^{2}\right] & =d+1-1 / \nu,
\end{aligned}
$$

(where $1 / \nu$ is the scaling dimension of the coupling conjugate to $\mathcal{O}=|\psi|^{2}$ ) we obtain

$$
\begin{aligned}
\operatorname{dim}\left[\chi_{T}\right] & =-d-1+2 \operatorname{dim}\left[T^{i 0}\right]=d+1, \\
\operatorname{dim}\left[\left\langle\Upsilon^{i}(-k,-\omega) \Upsilon^{i}(k, \omega)\right\rangle_{\mathrm{ret}}\right] & =-d-1+2 \operatorname{dim}\left[\Upsilon^{j}\right]=d+3-2 / \nu .
\end{aligned}
$$

Thus $\chi_{T} \sim T^{d+1}$, and

$$
\frac{1}{\tau_{\mathrm{imp}}} \sim V_{\mathrm{imp}}^{2} T^{d+1-2 / \nu},
$$

which is the result quoted in Eq. (1.11) for $d=2$. As noted there, $\nu \approx 2 / 3$, and so $1 / \tau_{\text {imp }}$ is roughly temperature independent.

An alternative, but less constructive argument proceeds along the lines of the discussion in Ref. 54. From Eq. (4.1) we have $\operatorname{dim}[V]=1 / \nu$, and so from Eq. (1.10) we have $\operatorname{dim}\left[V_{\text {imp }}^{2}\right]=$ $-d+2 / \nu$. This is indeed familiar from Harris' criterion which states that disorder is relevant if $\nu<2 / d$. Then, knowing $\operatorname{dim}\left[1 / \tau_{\mathrm{imp}}\right]=1$, the result (4.9) follows.

\section{DYONIC BLACK HOLES}

\section{A. $\mathbf{A d S}_{4} / \mathrm{CFT}_{3}$ and the black hole solution}

From the point of view of studying quantum critical phenomena, the AdS/CFT correspondence [55] provides a wealth of new solvable strongly correlated conformal field theories (CFTs) in $2+1$ dimensions. The key feature of these CFTs is that they admit a large $N$ limit in which they can be described classically as a gravitational theory in $3+1$ dimensions that asymptotes to Anti-de Sitter space $\left(\mathrm{AdS}_{4}\right)$. The CFT is thought of as living on the 'boundary' of the higher dimensional, or 'bulk', spacetime. 
The correspondence furthermore allows us to consider departures from criticality due to finite temperature. This is precisely the type of systems we are studying in this paper. Finite temperature in field theory corresponds to allowing the bulk spacetime to contain a black hole [56]. The temperature of the field theory is just the Hawking temperature of the black hole. Finite temperature dissipation in field theory is dual to bulk matter fields falling into the black hole.

We wish to consider a CFT with a global $U(1)$ symmetry and a corresponding charge density $\rho$ and a background magnetic field $B$. It was explained recently that this is dual to taking a dyonic black hole, carrying both electric and magnetic charge [29]. These black holes are solutions to Einstein-Maxwell theory in $3+1$ dimensions. In this section, and also in a separate paper [30], we will see how thermoelectric transport properties of the dyonic black hole precisely agree with our general analysis in the hydrodynamic limit. The black hole, via the AdS/CFT correspondence, provides a solvable example of the physics we are studying throughout this paper. Furthermore, the various particle-vortex dualities we have discussed above are seen to acquire a very transparent interpretation in the AdS/CFT correspondence.

The canonical example of the $\mathrm{AdS}_{4} / \mathrm{CFT}_{3}$ correspondence describes the infrared fixed point of maximally supersymmetric Yang-Mills theory with $S U(N)$ gauge group. The dual gravitational theory in this case is $M$ theory on $\mathrm{AdS}_{4} \times S^{7}$ [55]. However, in the large $N$ limit and for the subset of field theory questions we are asking, this theory may be consistently truncated to Einstein-Maxwell theory with a negative cosmological constant in $3+1$ dimensions. The dimensional reduction is performed for instance in [14].

The action for Einstein-Maxwell theory with a negative cosmological constant may be taken to be

$$
I=\frac{\sqrt{2} N^{3 / 2}}{6 \pi} \int d^{4} x \sqrt{-g}\left[-\frac{1}{4} R+\frac{1}{4} F_{\mu \nu} F^{\mu \nu}-\frac{3}{2}\right],
$$

which implies the equations of motion

$$
\begin{aligned}
R_{\mu \nu} & =2 F_{\mu \sigma} F_{\nu}{ }^{\sigma}-\frac{1}{2} g_{\mu \nu} F_{\sigma \rho} F^{\sigma \rho}-3 g_{\mu \nu}, \\
\nabla_{\mu} F^{\mu \nu} & =0 .
\end{aligned}
$$

We have expressed the normalization of the action in terms of the field theory quantity $N$.

A black hole in $A d S_{4}$ with planar horizon has metric

$$
d s^{2}=\frac{\alpha^{2}}{z^{2}}\left[-f(z) d t^{2}+d x^{2}+d y^{2}\right]+\frac{1}{z^{2}} \frac{d z^{2}}{f(z)} .
$$

The dyonic black hole carries both electric and magnetic charge

$$
F=h \alpha^{2} d x \wedge d y+q \alpha d z \wedge d t,
$$

where $h, q$ and $\alpha$ are constants that will be related to field theory quantities shortly. The function appearing in the metric is then

$$
f(z)=1+\left(h^{2}+q^{2}\right) z^{4}-\left(1+h^{2}+q^{2}\right) z^{3} .
$$


In these coordinates, the conformal boundary of the spacetime is at $z \rightarrow 0$ whereas the black hole event horizon is at $z=1$.

In the following subsection we summarize the thermodynamic properties of this black hole spacetime that were derived in [29], which is also the thermodynamics of the CFT. We will express thermodynamic quantities in terms of the dual field theory background magnetic field and chemical potential. These are related to the constants $q$ and $h$ that appeared in the black hole solution as follows [29]

$$
B=h \alpha^{2}, \quad \mu=-q \alpha .
$$

\section{B. The grand canonical ensemble}

We give the thermodynamics of the CFT in terms of the temperature $T$, the chemical potential $\mu$ and the background magnetic field $B$. Many variables are most simply expressed in terms of an auxiliary quantity $\alpha(T, \mu, B)$ which is determined from

$$
\frac{4 \pi T}{\alpha}=3-\frac{\mu^{2}}{\alpha^{2}}-\frac{B^{2}}{\alpha^{4}}
$$

The thermodynamic potential is

$$
\Omega=\frac{\sqrt{2} N^{3 / 2}}{6 \pi} \frac{\mathcal{V} \alpha^{3}}{4}\left(-1-\frac{\mu^{2}}{\alpha^{2}}+3 \frac{B^{2}}{\alpha^{4}}\right)
$$

Here $\mathcal{V}=\int d x d y$ is the spatial volume. The energy density is

$$
\varepsilon=\frac{\sqrt{2} N^{3 / 2}}{6 \pi} \frac{\alpha^{3}}{2}\left(1+\frac{\mu^{2}}{\alpha^{2}}+\frac{B^{2}}{\alpha^{4}}\right) .
$$

The entropy density is

$$
s=\frac{\sqrt{2} N^{3 / 2}}{6} \alpha^{2} .
$$

The charge density is

$$
\rho=\frac{\sqrt{2} N^{3 / 2}}{6 \pi} \alpha^{2} \frac{\mu}{\alpha} .
$$

The magnetization is

$$
M=-\frac{1}{\mathcal{V}} \frac{\partial \Omega}{\partial B}=-\frac{\sqrt{2} N^{3 / 2}}{6 \pi} \alpha \frac{B}{\alpha^{2}} .
$$

The pressure is

$$
P=M B+\frac{\varepsilon}{2} .
$$

These formulae satisfy the thermodynamic relation

$$
\varepsilon+P=T s+\mu \rho .
$$


Note that the above implies the relation

$$
\varepsilon=\frac{2}{3}(s T+\mu \rho-M B) .
$$

Finally, it is useful to define

$$
\chi=\frac{\sqrt{2} N^{3 / 2}}{6 \pi} T .
$$

Which gives the relation

$$
\rho M T^{2}=-\mu B \chi^{2}
$$

\section{Magnetization densities and currents}

In the following subsection, we will use Kubo formulae to the obtain transport coefficients of the SCFT from retarded Greens functions. When applying the Kubo formula to systems with a background magnetic field, it is necessary to subtract effects due to magnetization currents, as explained in Cooper et al. [43]. The magnetization currents are

$$
J_{i}^{\text {mag. }}=\epsilon_{i j} \partial_{j} M
$$

and

$$
T_{t i}^{\text {mag. }}=\epsilon_{i j} \partial_{j} M^{E} .
$$

Here and below the indices $i, j$ run over the two spatial coordinates $x$ and $y$. The equilibrium magnetization density $M$ and energy magnetization density $M^{E}$ for the dyonic black hole are obtained by differentiating the free energy with respect to a constant magnetic field for either the charge or momentum currents,

$$
\begin{aligned}
M & =-\frac{\delta \Omega}{\delta F_{x y}}, \\
M^{E} & =-\frac{\delta \Omega}{\delta F_{x y}^{E}} .
\end{aligned}
$$

Here we define $\delta F_{x y}^{E}=\partial_{x} \delta g_{t y}^{0}-\partial_{y} \delta g_{t x}^{0}$ and $\delta F_{x y}=\partial_{x} \delta A_{y}^{0}-\partial_{y} \delta A_{x}^{0}$, where $\delta g_{t a}^{0}$ is a background gauge field sourcing $T_{t a}$, and $\delta A_{a}^{0}$ sources $J_{a}$. Further comments on these magnetization densities, and their computation for the scalar field theory in Eq. (1.3) appear in Appendix B.

In the AdS/CFT correspondence, the free energy $\Omega$ is just the action of the dual gravitational background. To compute the derivatives in (5.20) and (5.21) we must consider on shell fluctuations of the bulk metric and gauge field that tend towards $\delta g_{t a}^{0}$ and $\delta A_{a}^{0}$, respectively, near the conformal boundary $z \rightarrow 0$. We then differentiate the action with respect to the boundary values of these fields.

More concretely, the boundary condition may be taken to be

$$
\begin{array}{r}
\delta A_{y} \rightarrow x B \quad \text { as } \quad z \rightarrow 0 \\
\delta g_{t y} z^{2} / \alpha \equiv \delta G_{y} \rightarrow x B^{E} \quad \text { as } \quad z \rightarrow 0
\end{array}
$$


with $B$ and $B^{E}$ constants and all other fields having normalizable behavior near the boundary. It turns out that the linearized fluctuation equations about the dyonic black hole background with these boundary conditions may be consistently truncated to the fields $\delta A_{y}$, $\delta A_{t}$ and $\delta g_{t y}$. The solution is

$$
\begin{aligned}
\delta A_{y} & =x\left(B-q B^{E} z\right), \\
\delta G_{y} & =x f(z) B^{E}, \\
\delta A_{t} & =\frac{h B^{E}}{2 \alpha}\left(z^{2}-1\right)-\frac{h B}{q \alpha}(z-1) .
\end{aligned}
$$

Note that $A_{t}$ vanishes at the horizon, $z=1$, as required.

Because we are fluctuating about a solution, the linear variation of the bulk action will vanish. However, there will be a boundary term that arises due to integration by parts when evaluating the action on the solution. There is also a boundary term that must be included to renormalize the gravitational action

$$
I_{\mathrm{bdy} .}=-\frac{\sqrt{2} N^{3 / 2}}{6 \pi}\left[\frac{1}{2} \int d^{3} x \sqrt{-\gamma} \theta+\int d^{3} x \sqrt{-\gamma}\right],
$$

where $\gamma$ is the boundary metric and $\theta=\gamma^{\mu \nu} \theta_{\mu \nu}$ is the trace of the extrinsic curvature $\theta_{\mu \nu}=-\frac{1}{2}\left(\nabla_{\mu} n_{\nu}+\nabla_{\nu} n_{\mu}\right)$, with $n$ an outward directed unit normal vector to the boundary.

For fluctuations about a solution, we have

$$
\delta I=\frac{\sqrt{2} N^{3 / 2}}{6 \pi} \int d^{3} x \sqrt{-\gamma}\left[-F_{\mu \nu} n^{\nu} \delta A^{\mu}+\frac{1}{4}\left(\theta^{\mu \nu}-\theta \gamma^{\mu \nu}-2 \gamma^{\mu \nu}\right) \delta g_{\mu \nu}\right] .
$$

Evaluated on the background (5.3) and (5.4), and considering only $\delta A_{y}, \delta A_{t}$ and $\delta g_{t y}$, this expression becomes

$$
\delta I=\frac{\sqrt{2} N^{3 / 2}}{6 \pi} q \alpha^{2} \int d^{3} x \delta A_{t} .
$$

Note that only the first term in the variation of the action (5.28) contributes. Using the solution (5.26) for $\delta A_{t}$, we obtain the magnetization

$$
M=-\frac{\delta S}{\delta B}=-\frac{\sqrt{2} N^{3 / 2}}{6 \pi} \alpha h=-\frac{\sqrt{2} N^{3 / 2}}{6 \pi} \frac{B}{\alpha},
$$

in complete agreement with our previous expression (5.12). For the energy magnetization we obtain

$$
M^{E}=-\alpha \frac{\delta S}{\delta B^{E}}=-\frac{q \alpha}{2} M=\frac{\mu M}{2} .
$$

\section{Transport coefficients in the d.c. limit}

We will obtain the transport coefficients using Kubo formulae for the retarded Greens functions. The Greens functions are obtained by considering fluctuations about the dyonic 
black hole background. In [29] these functions were obtained at $k=0$ and to leading order as $\omega \rightarrow 0$ with $B$ and $\rho$ held fixed. Unlike in our general magnetohydrodynamic (MHD) analysis in Section III, no assumptions are made here requiring $B$ to be small. The currentcurrent correlator is

$$
G_{J^{i} J^{j}}^{R}(\omega)=-\frac{\rho}{B} i \omega \epsilon_{i j},
$$

the current-momentum correlator is

$$
G_{J^{i} T^{t j}}^{R}(\omega)=-\frac{3 \varepsilon}{2 B} i \omega \epsilon_{i j}
$$

and the momentum-momentum correlator is

$$
G_{T^{t i} T^{t j}}^{R}(\omega)=-\frac{\chi T^{3} s^{2}}{\chi^{2} B^{2}+\rho^{2} T^{2}} i \omega \delta_{i j}-\frac{9 \rho \varepsilon^{2} T^{2}}{4 B\left(\chi^{2} B^{2}+\rho^{2} T^{2}\right)} i \omega \epsilon_{i j} .
$$

To relate these results to our general MHD study, we need an expression for the heat current $Q^{i}$. This is defined in Eq. (3.18); using the expression for the stress energy tensor in Eq. (3.24), we see that in linear response (small velocities with respect to the lab frame) we can work with

$$
Q^{i}=T^{t i}-\mu J^{i}
$$

leading to the correlators

$$
G_{Q^{i} J^{j}}^{R}(\omega)=\left(\frac{-s T}{B}+M\right) i \omega \epsilon_{i j}
$$

and

$$
G_{Q^{i} Q^{j}}^{R}(\omega)=-\frac{\chi T^{3} s^{2}}{\chi^{2} B^{2}+\rho^{2} T^{2}} i \omega \delta_{i j}+\frac{-\rho s^{2} T^{4}+B^{2} \mu^{2} \rho \chi^{2}+\rho T^{2} M^{2} B^{2}}{B\left(\chi^{2} B^{2}+\rho^{2} T^{2}\right)} i \omega \epsilon_{i j} .
$$

In obtaining these expressions, we used (5.15) and (5.17).

The electrical conductivity is given by the Kubo formula

$$
\sigma_{i j}=-\lim _{\omega \rightarrow 0} \frac{\operatorname{Im} G_{J^{i} J^{j}}^{R}(\omega)}{\omega}=\frac{\rho}{B} \epsilon_{i j} .
$$

The other thermoelectric tensors are also given by a Kubo formula. However, we should use the transport currents which are obtained from the supergravity currents by subtracting the magnetization currents [43]. The correct Kubo formula is

$$
\alpha_{i j}=-\frac{1}{T} \lim _{\omega \rightarrow 0} \frac{\operatorname{Im} G_{J^{i} Q^{j}}^{R}(\omega)}{\omega}+\frac{M}{T} \epsilon_{i j} .
$$

Thus we obtain

$$
\alpha_{i j}=\frac{s}{B} \epsilon_{i j}
$$

Similarly, the heat conductivity is given by the Kubo formula

$$
\bar{\kappa}_{i j}=-\frac{1}{T} \lim _{\omega \rightarrow 0} \frac{\operatorname{Im} G_{Q^{i} Q^{j}}^{R}(\omega)}{\omega}+\frac{2\left(M^{E}-\mu M\right)}{T} \epsilon_{i j},
$$


or

$$
\bar{\kappa}_{i j}=\frac{\chi T^{2} s^{2}}{\chi^{2} B^{2}+\rho^{2} T^{2}} \delta_{i j}+\frac{\rho s^{2} T^{4}}{T B\left(\chi^{2} B^{2}+\rho^{2} T^{2}\right)} \epsilon_{i j} .
$$

We can now compare these results with those of our general MHD computations, by taking the $\omega \rightarrow 0$ limit of the MHD transport coefficients (3.37). We see immediately that the expressions for $\sigma_{i j}$ and $\alpha_{i j}$ agree exactly. In order to match $\bar{\kappa}_{i j}$ we need to recall that the MHD results are only valid for small magnetic fields $B \ll T^{2}$, see Eq. (1.7). Furthermore, we need to know the conductivity $\sigma_{Q}$ for the dyonic black hole. It is shown in Ref. 30 that for the black hole

$$
\left.\sigma_{Q}\right|_{B=0}=\left(\frac{T s}{\varepsilon+P}\right)^{2} \frac{\chi}{T} .
$$

Using this formula and taking the small magnetic field limit, we obtain

$$
\left.\bar{\kappa}_{i j}\right|_{B \ll T^{2}}=\frac{\sigma_{Q}(\varepsilon+P)^{2}}{\rho^{2} T} \delta_{i j}+\frac{s^{2} T}{B \rho} \epsilon_{i j} .
$$

This expression now agrees exactly with the corresponding limit of the MHD result (3.37). Thus we see that the dyonic black hole fits into the general class of finite temperature deformations of quantum critical points that we have studied via hydrodynamics. The black hole gives specific values for $\sigma_{Q}$ and the other thermodynamic quantities and furthermore allows the results to be extended to arbitrary magnetic field.

\section{E. Bulk electromagnetic duality and CFT particle/vortex duality}

A consequence of the bulk description is that it gives a very transparent rationale behind the dualities in the transport coefficients that we commented upon earlier. The study of electromagnetic duality in the dyonic black hole is pursued in depth in Ref. 30, which furthermore obtains expressions for the black hole transport coefficients away from the d.c. limit. Here we shall summarize some results from that paper and show how they precisely match our expectations from MHD.

The bulk Maxwell theory enjoys an electromagnetic duality. This interchanges the bulk electric and magnetic fields $\boldsymbol{E} \rightarrow \boldsymbol{B}$ and $\boldsymbol{B} \rightarrow-\boldsymbol{E}$. Acting on the dyonic black hole solutions (5.3), this corresponds to $q \rightarrow h$ and $h \rightarrow-q$. Using (5.6) and (5.11) and the fact that the bulk coupling is inverted under electromagnetic duality, this implies that

$$
B \rightarrow \frac{T \rho}{\chi}, \quad \rho \rightarrow-\frac{T B}{\chi}, \quad \frac{\chi}{T} \rightarrow \frac{T}{\chi}
$$

Thus we see that the bulk electromagnetic duality maps the CFT into the same CFT with the values of the background magnetic field and charge density interchanged. This is the origin of the particle-vortex duality that we noted in our MHD computations. Indeed it is immediately seen that under the transformation (5.45) our expressions for $\sigma, \alpha$ and $\bar{\kappa}$ in Eqs. (5.38), (5.40) and (5.42) transform according to (3.42). There are some overall factors of $\chi / T$ 
different to (3.42), due to the fact that the transformation (5.45) is normalized differently to (3.40). The normalization in (5.45) is natural from the string perspective.

It remains to see how the thermoelectric transport coefficients of the black hole transform under this map. This is shown in detail in Ref. 30. The central point is the following. The bulk Maxwell potential $A$ determines the bulk electric and magnetic fields through $\boldsymbol{E} \sim \partial_{t} A$ and $\boldsymbol{B} \sim \partial_{z} A$. As we tend towards the boundary $z \rightarrow 0$, the electric piece is non-normalizable, and results in a boundary electric field $E$. The magnetic piece however is normalizable and therefore results in a boundary current $J$. The bulk electromagnetic duality is thus seen to exchange the electric field in the CFT with the current. More precisely, one finds

$$
E_{i} \leftrightarrow \epsilon_{i j} J_{j}
$$

As we commented below Eq. (3.42), this map together with the definition of the transport coefficients in Eq. (1.13) is enough to obtain all the duality transformations (3.42).

The results for the transport coefficients presented in the previous subsection were only obtained in the d.c. limit $\omega \rightarrow 0$ with $B$ and $\rho$ fixed, following Ref. 29. However, using the AdS/CFT dictionary, it is possible to study thermoelectric transport at all frequencies. This is done in Ref. 30. In particular, taking the limit $\omega \rightarrow 0$ with $\rho \sim B \sim T^{3 / 2} \sqrt{\omega}$, one obtains precisely the same expressions as those that followed from the MHD analysis (3.39), thus providing a consistency check for our picture. One can go further with the dyonic black hole and study transport and all $\rho, B$ and $\omega$ numerically. For all values, the particle-vortex duality holds automatically because of the bulk electromagnetic duality. This is the power of the AdS/CFT correspondence: all transport phenomena of the strongly correlated CFT at large $N$ are reduced to solving the equations for classical perturbations of the dual black hole in Einstein-Maxwell theory.

\section{CONCLUSIONS}

This paper has presented a general theory for hydrodynamic thermal and electric transport in in the vicinity of a quantum critical points described by "relativistic" quantum field theories. We have also shown that the results constitute a valuable starting point to understand experimental observations in a regime where no previous description was available.

It is perhaps useful to describe the results here from the vantage point of the Galileaninvariant hydrodynamic approaches which are traditionally used in condensed matter physics $[50,57]$. In such theories, charge (or number) currents are proportional to the momentum current, and consequently the conductivity is infinite in the absence of impurities (in the presence of a magnetic field, this implies Kohn's theorem [34]). The natural transport coefficient is the thermal conductivity, and this determines various diffusivities and damping constants.

In contrast, in the present paper, we have used a very different starting point. We 
considered a theory with both particle and anti-particle (hole) excitations, in which there is no proportionality between momentum and charge currents. For the case with particle-hole symmetry $(\rho=0)$ and $B=0$, the momentum and charge currents are decoupled from each other, and it is possible to have a charge current with no momentum current: the electrical current can decay to zero from such a state, and this decay is associated with the universal electrical conductivity $\sigma_{Q}[17]$. There is no analog to $\sigma_{Q}$ in the Galilean invariant case. Also, because of the symmetry of the stress-energy tensor, we could identify the energy current with the momentum density; the conservation of momentum then implied that the thermal conductivity was infinite [58]. Upon relaxing the requirement of particle-hole symmetry (i.e. allowing $\rho \neq 0$ ), we found the appearance of some characteristics of the Galilean invariant systems. In Eq. (1.2) we found that the excess particles (or holes) contributed a Drude-like conductivity above the quantum-critical $\sigma_{Q}$. The thermal conductivity became finite, but with a value related to $\sigma_{Q}$ by a Wiedemann-Franz-like relation. Finally, we also turned on a $B \neq 0$, and showed how all of the longitudinal and transverse transport co-efficients could be related to $\sigma_{Q}$ in relationships that were summarized in Section I.

While our analysis was specialized to relativistic quantum critical points, we expect that many aspects will generalize to other strongly interacting quantum critical points. Only a discrete particle-hole symmetry is required to decouple the charge and momentum currents, and this should be sufficient to obtain a finite $\sigma_{Q}$.

We also discussed applications of this general hydrodynamic structure to measurements of the Nernst co-efficient in the cuprates and NbSi films. For reasonable values of the parameters, we were able to reproduce several key aspects of the $B$ and $T$ dependence of the observations. Our results also make a significant prediction, characteristic of such "relativistic" theories of the superfluid-insulator transition: the presence of a hydrodynamic cyclotron mode. For the simplest case of a superfluid-insulator transition of Cooper pairs at integer filling as described by Eq. (1.3), this cyclotron mode can be considered due to the motion of Cooper pairs (or their dual vortices). However, for the more complicated examples at fractional filling noted in Section I A, such a simple interpretation is not possible, and the cyclotron mode is due to motion of all charge carriers, including those carrying fractional charges. From our fits to the data in the cuprates in Section II, we found that in presently studied samples this cyclotron mode was strongly overdamped by impurity scattering. However, this raises the possibility that the cyclotron resonance which might be observable in ultrapure samples. We estimated that the hydrodynamic cyclotron frequency in the cuprates was smaller than the cyclotron frequency of free electrons by a factor of order $10^{-3}$. Observation of this resonance would constitute a strong test of the theoretical ideas presented here, and we hope such experiments will be undertaken.

Another class of results in this paper described the remarkable holographic connection between this intricate hydrodynamic behavior in $2+1$ dimensions and the quantum theory of dyonic black holes in 3+1 dimensions. Using the AdS/CFT connection, we presented in Section $\mathrm{V}$ exact results for the hydrodynamic response functions of the vicinity of a $2+1$ 
dimensional supersymmetric conformal field theory. In the appropriate limiting regime, these results, and those in Ref. 30, were in complete agreement with those obtained from the magnetohydrodynamic analysis in Section III. This agreement lends strong support to the validity of our MHD analysis. Indeed, the analysis of the dual gravity theory helped guide our determination of the MHD equations.

\section{Acknowledgments}

Related results are reported in a companion paper by C. Herzog and one of us [30], and we thank C. Herzog for numerous valuable discussions. We acknowlege A. Vishwanath for pointing out that Eq. (2.4) agrees with the $T$ dependence of recent experimental data. We also thank P. W. Anderson, H. Aubin, M. J. Bhaseen, K. Behnia, A. G. Green, D. Podolsky, N. P. Ong, S. Shapiro, D. T. Son, S. L. Sondhi, A. Strominger, and A. Vishwanath for useful discussions. This research was supported by the NSF under grants PHY05-51164 (PK and SH) and DMR-0537077 (MM and SS) and by the Swiss National Fund for Scientific Research under grant PA002-113151 (MM).

\section{APPENDIX A: NORMAL MODES OF THE HYDRODYNAMIC EQUATIONS}

It is interesting to analyze the normal modes of the linearized magnetohydrodynamic equations in the absence of impurity scattering. Assuming a space and time dependence $e^{-i \omega t+i \vec{k} \cdot \vec{x}}$ of $\delta \mu, \delta T$ and $v_{\|}:=\vec{v} \cdot \vec{k} / k$ and $v_{\perp}:=(\vec{k} / k) \cdot(\hat{\epsilon} \vec{v})$, we find four independent modes satisfying the equations

$$
\begin{aligned}
\omega\left(\left.\frac{\partial \epsilon}{\partial \mu}\right|_{T} \delta \mu+\left.\frac{\partial \epsilon}{\partial T}\right|_{\mu} \delta T\right)-k(\epsilon+P) v_{\|} & =0 \\
\omega\left(\left.\frac{\partial \rho}{\partial \mu}\right|_{T} \delta \mu+\left.\frac{\partial \rho}{\partial T}\right|_{\mu} \delta T\right)-k\left(\rho v_{\|}+B \sigma_{Q} v_{\perp}\right)+i k^{2} \sigma_{Q}\left(\delta \mu-\frac{\mu}{T} \delta T\right) & =0 \\
\omega(\epsilon+P) v_{\|}-k(\rho \delta \mu+s \delta T)-i \rho B v_{\perp}+i B^{2} \sigma_{Q} v_{\|}-i k^{2}(\eta+\zeta) v_{\|} & =0 \\
\omega(\epsilon+P) v_{\perp}+k B \sigma_{Q}\left(\delta \mu-\frac{\mu}{T} \delta T\right)+i \rho B v_{\|}+i B^{2} \sigma_{Q} v_{\perp}-i k^{2} \eta v_{\perp} & =0 .
\end{aligned}
$$

In the long wavelength limit $k \ll 1$, one finds two modes corresponding to damped cyclotron oscillations of the plasma

$$
\omega_{ \pm}= \pm \omega_{c}-i \gamma
$$

These modes have a velocity field with $v_{\|}= \pm i v_{\perp}$, while $\delta \mu$ and $\delta T$ are smaller than $v_{\|}, v_{\perp}$ by a factor of order $\mathcal{O}(k)$.

Further there is a diffusive mode with frequency proportional to the conductivity $\sigma_{Q}$ and 
a quadratic dispersion relation

$$
\omega_{\mathrm{diff}}=-i \frac{k^{2} \sigma_{Q}(\epsilon+P)^{2}}{T\left(\left.\left.\frac{\partial \epsilon}{\partial \mu}\right|_{T} \frac{\partial \rho}{\partial T}\right|_{\mu}-\left.\left.\frac{\partial \epsilon}{\partial T}\right|_{\mu} \frac{\partial \rho}{\partial \mu}\right|_{T}\right)\left(\rho^{2}+B^{2} \sigma_{Q}^{2}\right)} .
$$

This mode has no fluctuations in energy density, $\delta \epsilon=\left.\frac{\partial \epsilon}{\partial \mu}\right|_{T} \delta \mu+\left.\frac{\partial \epsilon}{\partial T}\right|_{\mu} \delta T=0$. The velocity field $\vec{v}$ is of order $\mathcal{O}(k)$ relative to $\delta \mu, \delta T$.

Finally there is a subdiffusive, transverse shear mode with strongly suppressed fluctuations in temperature and longitudinal velocity component $\delta T=\mathcal{O}\left(k^{2}\right), v_{\|}=\mathcal{O}\left(k^{3}\right)$. It exhibits an unusual dispersion relation

$$
\omega_{\text {subdiff }}=\frac{i k^{4} \eta}{\left.B^{2} \frac{\partial \rho}{\partial \mu}\right|_{T}}
$$

and we have the relation

$$
i k \delta \mu \approx B v_{\perp}
$$

The origin of the $k^{4}$ dispersion (A4) can be seen as follows: A strongly suppressed $\omega(k)$ implies that momentum density is nearly conserved. Hence the total force density vanishes to lowest order, i.e., the Lorentz force is balanced by a longitudinal pressure gradient and a transverse friction force,

$$
\begin{aligned}
(\vec{J} \times \vec{B})_{\|} & \approx \vec{\nabla} P+\mathcal{O}(\eta, \zeta, \omega), \\
\eta \nabla^{2} \vec{v}_{\perp} & \approx(\vec{J} \times \vec{B})_{\perp}=-B J_{\|} .
\end{aligned}
$$

The first equation yields relation (A5). The second can be injected into the equation for charge conservation

$$
\partial_{t} \rho=-\vec{\nabla} \cdot \vec{J} \approx \vec{\nabla} \cdot\left[\frac{\eta}{B} \nabla^{2}\left(v_{\perp}\right)\right] \approx \vec{\nabla} \cdot\left[\frac{\eta}{B} \nabla^{2}\left(\frac{\vec{\nabla} \mu}{B}\right)\right]
$$

from which the dispersion follows upon using $\partial_{t} \rho=-\left.i \omega \frac{\partial \rho}{\partial \mu}\right|_{T} \delta \mu$.

\section{APPENDIX B: MAGNETIZATION AND ENERGY MAGNETIZATION}

In our computation of the transport co-efficients using the Kubo formula in Section V, we had to face the issue of the subtraction of magnetization currents, as discussed earlier in Refs. 42, 43. These subtractions were computed in Section V using the AdS/CFT mapping. This appendix describes the nature of these magnetization subtractions in the context of the scalar field theory in Eq. (1.3). Actually, most of the basic issues are already clarified in free 
field theory, and so we will limit our discussion here to this simple case. The generalization of the free field results to the interacting Wilson-Fisher fixed points can be straightforwardly carried out along the lines of Refs. 31, 32, and so we will not discuss it here.

So we consider here the free field version of Eq. 1.3 with Lagrangian

$$
\mathcal{L}=\left[\left(\partial^{\mu}+i A^{\mu}\right) \psi^{*}\right]\left[\left(\partial_{\mu}-i A_{\mu}\right) \psi\right]+m_{0}^{2}|\psi|^{2}
$$

The stress-energy tensor is [59]

$$
T_{\mu \nu}=\left[\left(\partial_{\mu}+i A_{\mu}\right) \psi^{*}\right]\left[\left(\partial_{\nu}-i A_{\nu}\right) \psi\right]+\left[\left(\partial_{\nu}+i A_{\nu}\right) \psi^{*}\right]\left[\left(\partial_{\mu}-i A_{\mu}\right) \psi\right]-g_{\mu \nu} \mathcal{L},
$$

while the $\mathrm{U}(1)$ current is

$$
J_{\mu}=i \psi^{*}\left(\partial_{\mu}-i A_{\mu}\right) \psi-i \psi\left(\partial_{\mu}+i A_{\mu}\right) \psi^{*}
$$

The equation of motion is

$$
\left(\partial_{\mu}-i A_{\mu}\right)\left(\partial^{\mu}-i A^{\mu}\right) \psi=m_{0}^{2} \psi
$$

It is now a straightforward, but tedious, exercise to verify that the above expressions do indeed imply the MHD equation of motion in Eqs. (3.1,3.2).

For the thermodynamics, we need the particle and hole eigenenergies. These are organized in Landau levels, with energy

$$
\epsilon_{\ell}^{2}=2 B(\ell+1 / 2)+m_{0}^{2}
$$

$(\ell=0,1, \ldots \infty)$ and degeneracy per unit area of $B /(2 \pi)$. From this, we can easily obtain expressions for the grand potential (for all thermodynamic quantities we subtract an infinite $T=0$ value, and $\omega_{n}$ is a Matsubara frequency which is an integer multiple of $2 \pi$ ):

$$
\begin{aligned}
\frac{\Omega}{\mathcal{V}}=-P & =\frac{B T}{2 \pi} \sum_{\omega_{n}} \sum_{\ell} \ln \left[\left(\omega_{n}-i \mu\right)^{2}+\epsilon_{\ell}^{2}\right] \\
& =\frac{B T}{2 \pi} \sum_{\ell}\left[\ln \left(1-e^{-\left(\epsilon_{\ell}-\mu\right) / T}\right)+\ln \left(1-e^{-\left(\epsilon_{\ell}+\mu\right) / T}\right)\right] .
\end{aligned}
$$

We also obtain the entropy, $s$, the density, $\rho$, and the magnetization density, $M$, by

$$
s=-\frac{1}{\mathcal{V}} \frac{\partial \Omega}{\partial T} ; \rho=-\frac{1}{\mathcal{V}} \frac{\partial \Omega}{\partial \mu} ; M=-\frac{1}{\mathcal{V}} \frac{\partial \Omega}{\partial B} .
$$

Following Cooper et al. [43], it is useful to define an internal pressure $P_{\text {int }}$ which equals

$$
\begin{aligned}
P_{\text {int }} & =P-M B \\
& =\frac{B^{2}}{2 \pi} \sum_{\ell} \frac{\ell+1 / 2}{\epsilon_{\ell}}\left[\frac{1}{e^{\left(\epsilon_{\ell}-\mu\right) / T}-1}+\frac{1}{e^{\left(\epsilon_{\ell}+\mu\right) / T}-1}\right] .
\end{aligned}
$$


We define the energy density $\varepsilon$ by $\left\langle T_{t t}\right\rangle$. Evaluating this from (B2) in Euclidean Matsubara space, we obtain

$$
\begin{aligned}
\varepsilon=\left\langle T_{t t}\right\rangle & =\frac{B}{2 \pi} \sum_{\ell} T \sum_{\omega_{n}} \frac{-\left(\omega_{n}-i \mu\right)^{2}+\epsilon_{\ell}^{2}}{\left(\omega_{n}-i \mu\right)^{2}+\epsilon_{\ell}^{2}} \\
& =\frac{B}{2 \pi} \sum_{\ell} \epsilon_{\ell}\left[\frac{1}{e^{\left(\epsilon_{\ell}-\mu\right) / T}-1}+\frac{1}{e^{\left(\epsilon_{\ell}+\mu\right) / T}-1}\right] .
\end{aligned}
$$

It is now easily verified that the relations in Eq. (3.15) are obeyed.

In a similar manner, we can compute $\left\langle T_{x x}\right\rangle$ and find

$$
\begin{aligned}
\left\langle T_{x x}\right\rangle & =\frac{B}{2 \pi} \sum_{\ell} T \sum_{\omega_{n}} \frac{-\left(\omega_{n}-i \mu\right)^{2}-m_{0}^{2}}{\left(\omega_{n}-i \mu\right)^{2}+\epsilon_{\ell}^{2}} \\
& =\frac{B^{2}}{2 \pi} \sum_{\ell} \frac{\ell+1 / 2}{\epsilon_{\ell}}\left[\frac{1}{e^{\left(\epsilon_{\ell}-\mu\right) / T}-1}+\frac{1}{e^{\left(\epsilon_{\ell}+\mu\right) / T}-1}\right] \\
& =P_{\mathrm{int}}
\end{aligned}
$$

and so Eqs. (3.10) are also obeyed.

Let us now write down the explicit result for $M$ from Eq. (B7):

$$
\begin{aligned}
M=-\frac{T}{2 \pi} \sum_{\ell}\left[\ln \left(1-e^{-\left(\epsilon_{\ell}-\mu\right) / T}\right)+\ln \left(1-e^{-\left(\epsilon_{\ell}+\mu\right) / T}\right)\right] \\
\quad-\frac{B}{2 \pi} \sum_{\ell} \frac{\ell+1 / 2}{\epsilon_{\ell}}\left[\frac{1}{e^{\left(\epsilon_{\ell}-\mu\right) / T}-1}+\frac{1}{e^{\left(\epsilon_{\ell}+\mu\right) / T}-1}\right] .
\end{aligned}
$$

A direct evaluation of $\langle\vec{r} \times \vec{J}\rangle$ in an infinite system yields only the second term but not the first. We now argue that the first term is the contribution of edge states. Notice that this first term can be rewritten in the form

$$
\begin{aligned}
M=\frac{1}{2 \pi} & \int_{0}^{\infty} d E g_{e}(E)\left[\frac{1}{e^{(E-\mu) / T}-1}+\frac{1}{e^{(E+\mu) / T}-1}\right] \\
& -\frac{B}{2 \pi} \sum_{\ell} \frac{\ell+1 / 2}{\epsilon_{\ell}}\left[\frac{1}{e^{\left(\epsilon_{\ell}-\mu\right) / T}-1}+\frac{1}{e^{\left(\epsilon_{\ell}+\mu\right) / T}-1}\right],
\end{aligned}
$$

where we can interpret $g_{e}(E)$ as the magnetization of edge states:

$$
g_{e}(E)=\left\{\begin{array}{ll}
0, & \text { for } E<\epsilon_{0} \\
\ell, & \text { for } \epsilon_{\ell-1}<E<\epsilon_{\ell}
\end{array} .\right.
$$

Note that $g_{e}(E)$ is a piecewise constant function, and there is one additional edge state as each Landau level is crossed, as expected. We can write the expression for the magnetization as a sum of a bulk and edge contributions as

$$
M=\frac{1}{2 \pi} \int_{0}^{\infty} d E g(E)\left[\frac{1}{e^{(E-\mu) / T}-1}+\frac{1}{e^{(E+\mu) / T}-1}\right],
$$


where

$$
g(E)=g_{e}(E)-\sum_{\ell=0}^{\infty} \frac{B(\ell+1 / 2)}{\epsilon_{\ell}} \delta\left(E-\epsilon_{\ell}\right) .
$$

With the above form for $M$, we can now immediately use the results of Ref. 42 (compare their Eqns. (31) and (34)) to obtain the value of $M^{E}$ :

$$
M^{E}=\frac{1}{2 \pi} \int_{0}^{\infty} d E E g(E)\left[\frac{1}{e^{(E-\mu) / T}-1}-\frac{1}{e^{(E+\mu) / T}-1}\right] .
$$

The subtraction for $\bar{\kappa}_{x y}$ is, by Cooper et al. [43] Eq. (69), $2 M^{Q}$ where

$$
\begin{aligned}
M^{Q} & =M^{E}-\mu M \\
& =\frac{1}{2 \pi} \sum_{\ell}\left[\int_{\epsilon_{\ell}-\mu}^{\epsilon_{\ell}+\mu} \frac{E d E}{e^{E / T}-1}-\frac{B(\ell+1 / 2)}{\epsilon_{\ell}}\left(\frac{\epsilon_{\ell}-\mu}{e^{\left(\epsilon_{\ell}-\mu\right) / T}-1}-\frac{\epsilon_{\ell}+\mu}{e^{\left(\epsilon_{\ell}+\mu\right) / T}-1}\right)\right]
\end{aligned}
$$

An alternative evaluation of $M^{Q}$, which is generalizable to interacting theories, follows from the representation of the heat current discussed in Ref. 60. As noted in Section V C, we need the response to a "magnetic field" which associated with the energy (or heat) current, just as the ordinary magnetization is a response to a magnetic field associated with the charge current. So we introduce a vector potential $\vec{A}_{Q}$ which couples to heat current: this is done by the replacement [60] $\vec{A} \rightarrow \vec{A}+i \vec{A}_{Q} \omega_{n}$. Consequently, the free energy density in the presence of this additional "magnetic field" $B_{Q}$ is obtained from Eq. (B6) simply by the replacement $B \rightarrow B+i B_{Q} \omega_{n}$, which yields

$$
\frac{\Omega}{\mathcal{V}}=\frac{T}{2 \pi} \sum_{\ell} \sum_{\omega_{n}}\left(B+i B_{Q} \omega_{n}\right) \ln \left[\left(\omega_{n}-i \mu\right)^{2}+2\left(B+i B_{Q} \omega_{n}\right)(\ell+1 / 2)+m_{0}^{2}\right] .
$$

Taking the derivative with respect to $B_{Q}$ we obtain

$$
\begin{aligned}
M^{Q}= & -\frac{1}{\mathcal{V}} \frac{\partial \Omega}{\partial B_{Q}} \\
= & \frac{T}{2 \pi} \sum_{\ell} \sum_{\omega_{n}}\left(-i \omega_{n} \ln \left[\left(\omega_{n}-i \mu\right)^{2}+\epsilon_{\ell}^{2}\right]+\frac{-2 i \omega_{n} B(\ell+1 / 2)}{\left(\omega_{n}-i \mu\right)^{2}+\epsilon_{\ell}^{2}}\right) \\
= & \frac{T}{2 \pi} \sum_{\ell} \sum_{\omega_{n}}\left(-i \omega_{n} \ln \left[-i \omega_{n}+\epsilon_{\ell}-\mu\right]-i \omega_{n} \ln \left[i \omega_{n}+\epsilon_{\ell}+\mu\right]\right) \\
& \quad+\frac{1}{2 \pi} \sum_{\ell}\left[-\frac{B(\ell+1 / 2)}{\epsilon_{\ell}}\left(\frac{\epsilon_{\ell}-\mu}{e^{\left(\epsilon_{\ell}-\mu\right) / T}-1}-\frac{\epsilon_{\ell}+\mu}{e^{\left(\epsilon_{\ell}+\mu\right) / T}-1}\right)\right] \\
= & \frac{1}{2 \pi} \sum_{\ell}\left[\int_{\epsilon_{\ell}-\mu}^{\epsilon_{\ell}+\mu} \frac{E d E}{e^{E / T}-1}-\frac{B(\ell+1 / 2)}{\epsilon_{\ell}}\left(\frac{\epsilon_{\ell}-\mu}{e^{\left(\epsilon_{\ell}-\mu\right) / T}-1}-\frac{\epsilon_{\ell}+\mu}{e^{\left(\epsilon_{\ell}+\mu\right) / T}-1}\right)\right],
\end{aligned}
$$

which agrees with Eq. (B17).

[1] Z. A. Xu, N. P. Ong, Y. Wang, T. Kakeshita, and S. Uchida, Nature 406, 486 (2000). 
[2] Y. Wang, L. Li, M. J. Naughton, G. D. Gu, S. Uchida, and N. P. Ong, Phys. Rev. Lett. 95, $247002(2005)$.

[3] L. Li, J. G. Checkelsky, S. Komiya, Y. Ando, N. P. Ong, Nature Physics 3, 311 (2007).

[4] Y. Wang, L. Li, and N. P. Ong, Phys. Rev. B 73, 024510 (2006).

[5] A. Pourret, H. Aubin, J. Lesueur, C. A. Marrache-Kikuchi, L. Bergé, L. Dumoulin, and K. Behnia, Nature Physics 2, 683 (2006).

[6] A. Pourret, H. Aubin, J. Lesueur, C. A. Marrache-Kikuchi, L. Bergé, L. Dumoulin, and K. Behnia, arXiv:cond-mat/0701376.

[7] V. Oganesyan and I. Ussishkin, Phys. Rev. B 70, 054503 (2004).

[8] I. Ussishkin, S. L. Sondhi, and D. A. Huse, Phys. Rev. Lett. 89, 287001 (2002).

[9] S. Mukerjee and D. A. Huse, Phys. Rev. B 70, 014506 (2004).

[10] D. Podolsky, S. Raghu, and A. Vishwanath, arXiv:cond-mat/0612096.

[11] P. W. Anderson, arXiv:0705.1174.

[12] M. J. Bhaseen, A. G. Green, and S. L. Sondhi, Phys. Rev. Lett. 98, 166801 (2007).

[13] S. Sachdev, Quantum Phase Transitions, Cambridge University Press, Cambridge (1999).

[14] C. P. Herzog, P. Kovtun. S. Sachdev, and D. T. Son, Phys. Rev. D 75, 085020 (2007).

[15] M. P. A. Fisher, G. Grinstein, and S. M. Girvin, Phys. Rev. Lett. 64, 587 (1990).

[16] X.-G. Wen and A. Zee, Int. J. Mod. Phys. B 4, 437 (1990).

[17] K. Damle and S. Sachdev, Phys. Rev. B 56, 8714 (1997).

[18] M. Troyer and S. Sachdev, Phys. Rev. Lett. 81, 5418 (1998).

[19] L. Balents, L. Bartosch, A. Burkov, S. Sachdev, and K. Sengupta, Phys. Rev. B 71, 144508 (2005).

[20] L. Balents, L. Bartosch, and S. Sachdev, Annals of Physics 321, 1528 (2006).

[21] M. P. A. Fisher, P. B. Weichman, G. Grinstein, and D. S. Fisher, Phys. Rev. B 40, 546 (1989).

[22] M. E. Peskin, Annals of Physics, 113, 122 (1978).

[23] C. Dasgupta and B. I. Halperin, Phys. Rev. Lett. 47, 1556 (1981).

[24] T. Senthil, A. Vishwanath, L. Balents, S. Sachdev, and M. P. A. Fisher, Science 303, 1490 (2004)

[25] L. Balents, L. Bartosch, A. Burkov, S. Sachdev, and K. Sengupta, Phys. Rev. B 71, 144509 (2005).

[26] C. Lannert, M. P. A. Fisher, and T. Senthil, Phys. Rev. B 63, 134510 (2001).

[27] L. Balents and S. Sachdev, Annals of Physics, in press, arXiv:cond-mat/0612220.

[28] C. P. Herzog, JHEP 0212, 026 (2002).

[29] S. Hartnoll and P. Kovtun, arXiv:0704.1160.

[30] S. Hartnoll and C. P. Herzog, arXiv:0706.3228.

[31] A. V. Chubukov, S. Sachdev, and J. Ye, Phys. Rev. B 49, 11919 (1994); S. Sachdev, Phys. Lett. B 309, 285 (1993).

[32] S. Sachdev, Z. Phys. B 94, 469 (1994). 
[33] V. Oganesyan and I. Ussishkin, Phys. Rev. B 70, 054503 (2004).

[34] W. Kohn, Phys. Rev. 123, 1242 (1961).

[35] M. P. A. Fisher and G. Grinstein, Phys. Rev. Lett. 60, 208 (1988).

[36] J. Ye, Phys. Rev. B 58, 9450 (1998).

[37] A. V. Balatsky and P. Bourges, Phys. Rev. Lett. 82, 5337 (1999).

[38] N. Doiron-Leyraud, C. Proust, D. LeBoeuf, J. Levallois, J.-B. Bonnemaison, R. Liang, D. A. Bonn, W. N. Hardy, and L. Taillefer, Nature 447, 565 (2007).

[39] A. Larkin and A. Varlamov, Theory of Fluctuations in Superconductors Clarendon, Oxford (2005).

[40] Yu. N. Obraztsov, Fiz. Tverd. Tela 6, 414 (1964) [Sov. Phys.Solid State 6, 331 (1964)]; 7, 455 (1965).

[41] P. S. Zyryanov and V. P. Silin, Phys. Met. Metallogr. (USSR) 17, 130 (1964).

[42] H. Oji and P. Streda, Phys. Rev. B 31, 7291 (1985).

[43] N. R. Cooper, B. I. Halperin, and I. M. Ruzin, Phys. Rev. B 55, 2344 (1997).

[44] S. R. de Groot The Maxwell equations: Non-relativistic and relativistic derivations from electron theory, North-Holland Pub. Co., Amsterdam (1969).

[45] M. Demianski, Relativistic Astrophysics, Pergamon Press, Oxford (1985), Section 2.5.

[46] L. D. Landau and E. M. Lifshitz, Fluid Mechanics, Butterworth-Heinemann, Oxford (1987), Section 127.

[47] D. T. Son and A. O. Starinets, JHEP 0603, 052 (2006).

[48] L. D. Landau and E. M. Lifshitz, The Classical Theory of Fields, Butterworth-Heinemann, Oxford (1987), Section 33.

[49] B. I. Halperin and P. C. Hohenberg, Rev. Mod. Phys. 49, 435 (1977).

[50] L. P. Kadanoff and P. C. Martin, Annals of Physics, 24, 419 (1963).

[51] C. W. Misner, K. S. Thorne, and J. A. Wheeler, Gravitation, W. H. Freeman, San Francisco, (1973), Chapter 22.

[52] D. Forster, Hydrodynamic Fluctuations, Broken Symmetry, and Correlation Functions, Benjamin-Cummings, Reading (1975), Chapter 5.

[53] T. Giamarchi, Phys. Rev. B 44, 2905 (1991).

[54] M. Vojta, C. Buragohain, and S. Sachdev, Phys. Rev. B 61, 15152 (2000).

[55] J. M. Maldacena, Adv. Theor. Math. Phys. 2, 231 (1998) [Int. J. Theor. Phys. 38, 1113 (1999)] [arXiv:hep-th/9711200].

[56] E. Witten, Adv. Theor. Math. Phys. 2, 505 (1998) [arXiv:hep-th/9803131].

[57] G. Baym and C. Pethick, Landau Fermi-Liquid Theory: Concepts and Applications, WileyInterscience, New York (1991).

[58] M. Vojta, Y. Zhang, and S. Sachdev, Int. J. Mod. Phys. B 14, 3719 (2000); T. Senthil (unpublished).

[59] S. W. Hawking and G. F. R. Ellis, The large scale structure of space-time, Cambridge Univer- 
sity Press, Cambridge (1973).

[60] J. Moreno and P. Coleman, arXiv:cond-mat/9603079. 